\title{
Analyse et formalisation de la dimension durable du contrat didactique
}

Cas de l'enseignement de la physique par activités en classe de seconde Analysis and formalization of the perennial dimension of the didactic contract Case of the activity-based physics teaching in grade 10

\section{Patrice Venturini et Andrée Tiberghien}

\section{OpenEdition}

Journals

Édition électronique

URL : http://journals.openedition.org/educationdidactique/3539

DOI : ERREUR PDO dans /localdata/www-bin/Core/Core/Db/Db.class.php L.34 : SQLSTATE[HY000]

[2006] MySQL server has gone away

ISSN : 2111-4838

Éditeur

Presses universitaires de Rennes

Édition imprimée

Date de publication : 27 décembre 2018

Pagination : 65-106

ISBN : 978-2-7535-7774-9

ISSN : $1956-3485$

\section{Référence électronique}

Patrice Venturini et Andrée Tiberghien, «Analyse et formalisation de la dimension durable du contrat didactique ", Éducation et didactique [En ligne], 12-3 | 2018, mis en ligne le 01 janvier 2021, consulté le 08 janvier 2021. URL : http://journals.openedition.org/educationdidactique/3539; DOI : https:// doi.org/10.4000/educationdidactique.3539 


\title{
ANALYSE ET FORMALISATION DE LA DIMENSION DURABLE DU CONTRAT DIDACTIQUE CAS DE L'ENSEIGNEMENT DE LA PHYSIQUE PAR ACTIVITÉS EN CLASSE DE SECONDE
}

\author{
Patrice Venturini \\ UMR EFTS, Université de Toulouse Jean-Jaurès, France \\ Andrée Tiberghien \\ UMR ICAR, LABEX ASLAN, CNRS-Université de Lyon, France
}

\begin{abstract}
Cet article propose à la fois une méthode d'analyse du contrat didactique prenant en compte différentes échelles de temps et le résultat obtenu dans le cas d'une séquence d'enseignement en classe de seconde en physique. Le point de vue d'une action conjointe professeur - élèves et les concepts qui y sont traditionnellement associés, notamment contrat et milieu, transaction et jeu didactiques sont mobilisés dans l'article, le concept de jeu étant méthodologiquement associé à l'échelle mésoscopique de 10 à 30 minutes. Un point de vue théorique adapté de travaux en sciences du langage est utilisé à l'échelle microscopique de quelques secondes, basé sur le concept d'acte. Une échelle intermédiaire assure le lien entre niveaux microscopique et macroscopique. Des analyses développées à chacun de ces niveaux font apparaître des régularités dans l'action conjointe qui sont interprétées en fonction du contexte donné par les niveaux supérieurs. Elles permettent de formuler un certain nombre de règles formalisant en particulier les attentes réciproques du professeur et des élèves, d'abord dans une des séances analysées, puis sur l'ensemble de la séquence, à chacune des échelles. Ces analyses conduisent aussi à dégager deux échelles de temps supplémentaires ( $1 \mathrm{~h}$ à $1 \mathrm{~h} 30$ et 2 à $3 \mathrm{~h}$ ) dont la validité sur des corpus différents reste à montrer.
\end{abstract}

Mots-clés : contrat didactique, méthodologie, didactique de la physique.

Analysis and formalization of the perennial dimension of the didactic contract Case of the activity-based physics teaching in grade 10

This paper develops both a method to analyze the didactic contract involving different timescales and the description of the contract associated to a teaching sequence in physics education at grade 10. In our analyses we use the joint action theory in didactics and its usual concepts (contract, milieu, transaction and game), and we chose to associate the concept of didactic game to the mesoscopic scale (10 to 30 minutes). A viewpoint adapted from linguistics completes our theoretical framework at the microscopic level of some seconds, based on the concept of "act". An intermediary scale (1 to 5 minutes) bridges the microscopic and mesoscopic levels. We developed analyses at each timescale; they show regularities in the teacher and students joint action that we interpret according to their context, given by higher timescales. These regularities enabled us to formulate rules at different timescales, formalizing the reciprocal expectations of the teacher and the students, that is to say belonging to the didactic contract. These analyses lead us to establish two more timescales $(1 \mathrm{~h}-1 \mathrm{~h} 30 ; 2 \mathrm{~h}$ $3 h)$; their relevance must be checked on other kind of teaching sequences.

Keywords : didactic contract, methodology, physics education. 
Cet article porte sur l'analyse et la formalisation du contrat didactique dans un enseignement de la physique en classe de seconde. Son enjeu scientifique est essentiellement méthodologique et à un degré moindre théorique. En effet, la méthode que nous développons ici n'est pas fondée sur l'identification classique de ruptures dans le contrat, mais sur une émergence de significations résultant de l'articulation d'analyses développées à des échelles temporelles différentes, en lien avec des outils théoriques adaptés à ces échelles ${ }^{1}$. Parce que le contrat analysé est relatif à un enseignement par activité donnant une position centrale au travail des élèves et qu'il décrit des pratiques possibles d'enseignement de la physique, l'article a aussi des enjeux institutionnels et sociaux, mais ces enjeux sont conjoncturels à la séquence choisie pour l'analyse et ne constituent pas le focus de l'article.

L'étude menée nécessite bien sûr une analyse de l'action didactique, si bien que nous présentons d'abord les outils que nous mobilisons à cette fin, ils nous permettront aussi de problématiser la question de recherche. Classiquement, nous décrivons ensuite la méthode que nous avons développée pour expliciter les règles implicites ou non qui rendent compte du fonctionnement d'une classe de physique au lycée. L'explicitation de ces règles à diverses échelles de temps constitue l'essentiel de cet article et occupe les trois sections suivantes, la discussion de la méthode utilisée et des résultats obtenus terminant l'article.

\section{OUTILS THÉORIQUES}

Selon Nespor (2004, p. 309-310), l'enseignement et l'apprentissage incluent des « complex layered processes in which actions and events are simultaneously produced and made meaningful at multiple spatio-temporal scales ». Aussi, l'analyse de ces processus nécessite de prendre en compte plusieurs échelles de temps, l'échelle spatiale étant souvent limitée à la classe. De manière générale, les études didactiques distinguent trois échelles : une échelle macroscopique correspondant à la durée de l'observation; une échelle mésoscopique allant de 10 à 30 minutes environ, que nous considérons comme centrale dans les analyses car correspondant au rythme de changement des activités dans une classe; une échelle microscopique de l'ordre de la seconde (Tiberghien, Buty, Mortimer, Souassy, \& Mortimer,
2007 p. 102). Nous avons montré (Tiberghien et Venturini, 2015; Venturini et Tiberghien, 2012) l'utilité pour des raisons méthodologiques, d'une échelle intermédiaire de 1 à 5 minutes entre les niveaux microscopique et mésoscopique. Nous présentons en conséquence les outils théoriques que nous mobilisons à ces différentes échelles, d'abord mésoscopique (Niveau N), ensuite microscopique (Niveau N-2) et enfin intermédiaire entre les deux (Niveau $\mathrm{N}-1$ ), avec en préalable, un focus particulier sur la notion de contrat didactique dont la caractérisation fait l'objet de cet article, et qui impacte toutes les échelles temporelles.

\section{Contrat didactique}

Nous situons le contrat didactique dans le cadre de la théorie de l'action conjointe en didactique (TACD) (Sensevy et Mercier, 2007, Sensevy 2011, 2014), qui relève d'une approche pragmatique. Nous considérons ainsi que l'action d'enseigner du professeur et celle d'étudier ou d'apprendre de l'élève sont nécessairement conjointes. Cette action particulière, appelée action didactique, qui est par essence communicationnelle, est vue comme mettant en jeu une série de transactions entre le professeur et l'élève dont l'objet est un savoir ou est en lien avec un savoir. Le professeur, l'élève et le savoir constituent selon ce point de vue un ensemble ne pouvant être dissocié, car sinon le sens même de l'action didactique est perdue.

Dans un groupe classe donné, les acteurs, professeur et élèves, mettent en jeu des stratégies pour réaliser les transactions, stratégies prenant en compte les attentes réciproques des acteurs. En d'autres termes, il y a un contrat, plus ou moins implicite, sur lequel s'appuient ces transactions, celles-ci contribuant à son renforcement ou à son évolution. Ce contrat peut varier selon les transactions, et notamment suivant le type de savoir en jeu ; cependant le professeur et les élèves ne modifient pas leur façon de communiquer et de collaborer pour le traitement du savoir en jeu à chaque transaction ; aussi, certaines composantes du contrat vont être plus pérennes que d'autres dans la classe (Brousseau, 1997). Nous distinguons ainsi les composantes que nous appelons «durables » (par exemple l'habitude de travailler en groupe, celle d'argumenter ses réponses) des composantes plus conjoncturelles à 
une situation et en particulier à l'objet de la transaction qui, la plupart du temps, est du savoir.

Les transactions se réalisent dans un environnement spécifique matériel, conceptuel et social auquel les acteurs contribuent. Chacun utilise des éléments de cet environnement au regard du sens qu'il leur attribue dans la transaction en cours. Ces éléments de l'environnement qu'un acteur utilise constituent son milieu. Ainsi, le cadre théorique de la TACD que nous reprenons comporte les concepts d'action didactique conjointe, de contrat et de milieu qui sont associées aux transactions et qui permettent de rendre compte de l'enseignement et l'étude d'un savoir dans une classe. Ils ne sont liés ni à une échelle temporelle spécifique, ni à « l'étendue » du savoir en jeu. En revanche, les concepts de contrat et de milieu sont dépendants du temps : en effet, le système social dans lequel la classe est insérée demande que le savoir évolue au cours du temps d'enseignement. Notre cadre théorique reprend les concepts qui permettent de rendre compte de l'évolution temporelle du savoir, la chronogenèse, de l'évolution des responsabilités prises dans le déroulement de transactions, la topogenèse, et de celle du milieu, la mésogenèse. Nous précisons maintenant comment nous mettons en ouvre ces trois concepts aux différentes échelles. Ils correspondent en effet à trois dimensions de l'action didactique, et les dynamiques dont ils rendent compte sont nécessairement en relation avec le contrat didactique.

\section{Outils théoriques mobilisés à l'échelle mésoscopique (Niveau $\mathrm{N}$ )}

Si, dans la TACD, l'action conjointe peut être vue comme une série de transactions, elle peut aussi être vue comme une série de jeux, ces deux métaphores n'étant pas sans lien. La première met l'accent sur ce que professeur et élève co-construisent au cours des échanges et dont l'aboutissement servira de référence commune pour les transactions à venir. La seconde met plus l'accent sur des aspects qui restent souvent implicites dans la transaction mais qui la pilotent, les idées de règles, de stratégies, d'enjeu, de gain. C'est la raison pour laquelle, dans la suite, nous mobilisons plus la notion de jeu. Cette perspective renvoie au « jeu social » de Bourdieu (Bourdieu, 1992) et au « jeu de langage » de Wittgenstein (Wittgenstein et Anscombe, 1997). Un jeu se définit par un enjeu et par les règles pour y jouer. Aussi, on retrouve dans le jeu didactique un enjeu, le savoir, et des règles, qui relèvent du contrat et déterminent en partie l'évolution du milieu.

Tant la métaphore de la transaction que celle du jeu n'impliquent pas de temporalité particulière. Nous faisons le choix méthodologique d'associer le jeu (et la transaction) à l'échelle mésoscopique pouvant aller de quelques minutes à quelques dizaines de minutes, en lien avec le rythme habituel de changement de situation de travail dans la classe. Ainsi une séance d'enseignement va être structurée en jeux. Il y a changement de jeu quand l'enjeu, le contrat et/ou le milieu évoluent significativement, c'est-à-dire quand une ou plusieurs des trois dimensions de l'action didactique est modifiée. L'empan temporel que nous avons choisi pour le jeu détermine la prise en compte du caractère significatif ou non de l'évolution qui est continuelle et constitutive de l'action didactique.

\section{Outils théoriques mobilisés à l'échelle microscopique (Niveau N-2)}

Les concepts utiles pour caractériser l'action didactique à l'échelle mésoscopique renvoient à des dynamiques qui ne sont pas du tout perceptibles à l'échelle microscopique. Il est donc nécessaire d'utiliser des descripteurs adaptés à cette échelle qui est celle de l'événement de courte durée comme celle d'un acte de langage. Ces descripteurs doivent être à la fois cohérents avec le point de vue pragmatique de la TACD mobilisée au niveau mésoscopique et opérationnels pour les analyses.

L'action didactique est considérée dans la TACD comme " organiquement communicationnelle ", (Sensevy, 2011, p. 98). Aussi, nous avons emprunté au courant pragmatique des sciences du langage et plus particulièrement à Charaudeau (2004), à la fois l'idée de l'existence d'un lien étroit entre action et langage dans une perspective communicationnelle, et l'idée que ce lien pouvait être considéré selon trois points de vue, pragmatique, interactionnel et représentationnel (p. 161). Cette approche, qui envisage les rapports entre action et langage, nous permet d'étudier le niveau microscopique de l'action conjointe. Ainsi, à cette échelle, notre modèle (cf. Tiberghien et Venturini, 2015) caractérise des « actes » dont la plupart sont des actes de langage, 
à l'aide des points de vue proposés par Charaudeau, pragmatique, interactionnel et représentationnel, sans pour autant les interpréter indépendamment les uns des autres. Cet emprunt à une théorie linguistique que nous avons adaptée à notre contexte nous paraît compatible avec la TACD. En effet, cette approche linguistique partage avec la TACD un point de vue pragmatique en posant le nouage entre but de l'action et visée de la communication.

\section{L'acte, unité d'analyse au niveau microscopique}

L'acte que nous considérons comme le plus petit évènement gardant un sens du point de vue de la transaction didactique, constitue notre unité d'analyse microscopique. Sa durée est de l'ordre de la seconde. Nous considérons l'acte selon le point de vue de Mead, compatible avec le point de vue d'une action didactique conjointe : « the unit of existence is the act, not the moment. And the act stretches beyond the stimulus to the response. While most of our acts stretch into the world that does not yet exist, they inevitably include immediate steps which lie within the existent world " (Mead 1938, p. 65). Toutefois, la définition de l'acte que nous avons donnée ne donne pas de critères directement opératoires pour le découpage des transactions, laissant pour cela une grande part à l'interprétation et à l'intuition du chercheur, d'autant que comme le souligne Kerbrat-Orecchioni (1995), « différents actes peuvent non seulement se succéder dans un même énoncé, mais aussi s'y amalgamer » (p. 7).

\section{La dimension pragmatique}

Cette dimension est reliée aux forces illocutoire et perlocutoire du langage et rattachée à l'intentionnalité du professeur ou des élèves, en référence aux nombreux travaux sur les actes de langage (voir par exemple Searle, 1969 ; Searle \& Vanderveken, 1985). Cependant comme l'analyse Kerbrat-Orecchioni (1995), la multiplicité des taxinomies d'actes de langage nous a conduits à choisir nos propres catégories de descripteurs, établies de manière inductive et adaptées à notre propos qui est d'étudier l'évolution du savoir dans la classe. Ainsi d'un point de vue pragmatique, nous distinguons:

- les actes en lien direct avec les savoirs, généralement les plus nombreux dans une classe et bien sûr importants dans le contexte d'une analyse didactique. Au sein de cette catégorie, en prenant en compte la responsabilité exercée vis-à-vis du savoir, nous considérons :

- d'une part, les actes dans lesquels l'acteur, professeur ou élève, agit lui-même sur le savoir ; l'acte peut relever de la proposition (formulation d'assertions soumises à la discussion ou à l'évaluation, reformulation, complément, justification etc.), de l'information établie (savoirs, précisions conjoncturelles liées à la tâche), de l'évaluation des propositions (validation, jugement, etc.), ou d'autres types d'actes;

- d'autre part, les actes dans lesquels l'acteur demande à son partenaire d'agir sur le savoir ; la demande peut porter sur une proposition, une information, ou sur autre chose.

- les actes relatifs à la gestion de la classe en particulier à la communication concernant aussi bien la mise au travail des élèves que la distribution de la parole, ou encore la gestion des documents distribués par exemple ;

- des actes concernant l'aspect affectif (valorisation du travail, mise en confiance etc.) ; - les autres types d'actes (ce descripteur n'a pas été utilisé dans cette étude).

\section{La dimension interactionnelle}

À l'école, un acte de langage contribue aussi bien à un discours interactif que non-interactif, au sens de Mortimer et Scott (2000).

En empruntant des descripteurs aux travaux notamment à Sinclair et Coulthard $(1975,1992)$ et Chinn (2006), en fonction aussi de nos propres travaux empiriques, nous distinguons ici les actes correspondant à une question ou une injonction, ceux correspondant à une réponse, à une poursuite évaluative (évaluation succédant à une réponse) ou encore à une déclaration (assertion qui ne relève pas d'une réponse à une question).

\section{La dimension représentationnelle}

Cette dimension est liée à la représentation du monde physique. Trois descripteurs sont utilisés pour la caractériser : la facette de connaissance associée à l'acte identifié (ou à plusieurs), la caractéris- 
Patrice Venturini E Andrée Tiberghien

tique de cette facette et la tâche épistémique que sa production met en jeu.

Selon Minstrell (1992) et Galili et Hazan (2000), une facette est un énoncé mettant en jeu un élément de connaissance, de raisonnement ou de stratégie qui peut être correct ou incorrect du point de vue du savoir enseigné et relever alors par exemple de pratiques quotidiennes. Une facette est construite par le chercheur et sert de référence pour analyser le discours de la classe (professeur ou élève). Un élément de ce discours relève d'une facette quand le chercheur considère qu'il a le même sens que l'énoncé de la facette. Il y a donc un catalogue de facettes pour chaque corpus.

La facette est caractérisée de la manière suivante : - la manière dont la facette se situe par rapport à la modélisation : elle peut relever du monde des théories et modèles ou de celui des observables (objets et évènements) ou encore elle peut mettre en relation ces deux mondes (Tiberghien \& Malkoun, 2007) ;

- le type de langue quotidienne ou scientifique dont la facette relève ;

- la catégorie thématique à laquelle elle appartient (dans cette étude, le catalogue des facettes est organisé thématiquement).

Enfin, la production de la facette par un des acteurs, professeur ou élève, met en jeu une tâche épistémique c'est-à-dire à une opération de pensée (Ohlsson, 1996) qui peut-être une description empirique ou théorique, une interprétation, ou encore une prédiction, une mesure, une argumentation ou une comparaison etc. Signalons qu'une tâche épistémique peut être en jeu dans un seul acte (une prédiction, une mesure par exemple), ou plus fréquemment dans plusieurs (une argumentation, une interprétation par exemple). De plus, tous les actes ayant le savoir pour objet ne contribuent pas à des tâches épistémiques comme on pourra le voir dans le Tableau 2.

Notons dès à présent que notre grille d'analyse présentée dans la partie « Méthodologie » rassemble l'ensemble des modalités mobilisées dans l'étude pour chacune des dimensions (pragmatique, interactionnelle, représentationnelle).

\section{Outils théoriques mobilisés au niveau N-1}

En tant qu'intermédiaire entre les niveaux mésoscopique et microscopique, un épisode de niveau N-1 peut être considéré de deux manières différentes et complémentaires :

- comme la plus petite unité de transaction ou de jeu (en dessous, il n'y a plus de transaction ni de jeu). À ce titre, cet épisode peut être caractérisé à l'aide des descripteurs utilisés au niveau mésoscopique pour décrire l'action didactique et est lié à des évolutions « locales » d'une ou plusieurs de ses dimensions ;

- comme un ensemble d'actes, cohérent du point de vue de la signification, et à ce titre l'épisode de niveau N-1 peut être décrit à partir des caractéristiques des actes.

\section{Vue synoptique des outils théoriques}

Cet ensemble d'outils, dont le Tableau 1 donne une vue synoptique, permet de rendre compte de l'action didactique à différentes échelles et nous allons mettre à profit ses caractéristiques pour expliciter les règles souvent implicites mobilisées dans le fonctionnement d'une classe.

\section{PROblémATIQUe}

Le contrat didactique, considéré comme un système stratégique incluant notamment un ensemble de règles, le plus souvent implicites, mobilisées par les acteurs pour mener à bien des transactions dans une dialectique « milieu - contrat» (Sensevy, 2011), est un concept intéressant pour caractériser des pratiques d'enseignement et d'étude. Toutefois, dans les études qui sont consacrées à ces pratiques, le contrat fait l'objet de peu de descriptions systématiques. Certaines de ses composantes sont identifiées à la lumière de ses ruptures (par exemple Ligozat, Lundqvist, Amade-Escot, 2018) mais cette identification reste conjoncturelle à une situation donnée, et souvent centrée sur les composantes spécifiques au savoir en jeu. La dimension « durable » du contrat qui constitue l'arrière-plan des transactions développées dans la classe, et particulièrement, les règles qui relèvent de la nature et de la gestion des transactions, sont peu investiguées, ou tout au moins de manière systématique, sauf à considérer les travaux 
Tableau 1 : Récapitulatif des outils théoriques mobilisés aux différentes échelles

\begin{tabular}{|c|c|}
\hline Échelles & Outils théoriques \\
\hline Échelle mésoscopique (Niveau N) & $\begin{array}{l}\text { Structuration en jeux (transactions) didactiques (« épisodes } \mathrm{N} \text { »); } \\
\text { Pour chaque jeu : évolutions du savoir, des responsabilités prises dans le déroulement de la } \\
\text { transaction et du milieu au cours du temps }\end{array}$ \\
\hline Échelle intermédiaire (Niveau N-1) & $\begin{array}{l}\text { Structuration en «épisodes N-1 » : la petite unité de transaction ou de jeu ; } \\
\text { Pour chaque épisode N-1 : } \\
\text { - évolutions locales du savoir, des responsabilités prises dans le déroulement de transac- } \\
\text { tion et du milieu au cours du temps } \\
\text { - et/ou dimensions pragmatiques, interactionnelles et représentationnelles des actes qui } \\
\text { constituent correspondants, prises en compte de manière simultanée. }\end{array}$ \\
\hline Échelle microscopique (Niveau N-2) & $\begin{array}{l}\text { Structuration en actes ; } \\
\text { Pour chaque acte : caractérisation à l'aide des descripteurs suivants relevant des dimensions : } \\
\text { - pragmatique : acte mettant en ouvre } \\
\quad \text { - le savoir : proposition, évaluation, information, ... faite par l'acteur lui-même ou } \\
\quad \text { demandée à ses partenaires de l'action conjointe ; } \\
\text { - la gestion de classe ou de la communication, l'affectif... } \\
\text { - interactionnelle : question, injonction, réponse, poursuite évaluative, déclaration ; } \\
\text { - représentationnelle : facette du savoir caractérisée par son lien avec la modélisation, } \\
\text { le type de langue, la catégorie thématique dont elle relève et la tâche épistémique éven- } \\
\text { tuellement associée. }\end{array}$ \\
\hline
\end{tabular}

comme ceux de Cobb et ses collègues (McClain, \& Cobb, 2001 ; Yackel \& Cobb, 1996 ; Cobb, Stephan et McClain, 2001) sur l'identité normative. Ils ont en effet étudié systématiquement les « normes » d'une classe de mathématiques au sens où celles-ci définissent le bon élève dans cette classe. Nous proposons donc dans cette étude une analyse systématique de la dimension durable du contrat ; celle-ci nécessite la mise au point d'une méthode d'investigation nouvelle et particulière.

Aussi, il est souhaitable autant que faire se peut de se placer dans un contexte favorable pour la mener à bien. Reprenant la constatation que des règles du contrat en général implicites sont plus facilement identifiables lors de ruptures, nous avançons l'idée qu'un nouveau format d'enseignement introduit dans la classe est une situation favorable à nos desseins. Parallèlement, ce nouveau format ne doit pas être introduit ponctuellement, il doit avoir une certaine récurrence dans un espace temporel relativement réduit pour que de nouvelles composantes du contrat s'établissent.

L'enseignement de la physique comporte des modalités classiques (cours dialogués, travaux pratiques par exemple), des modalités plus récentes, la démarche d'investigation, l'enseignement par activités ou encore la résolution de problèmes. L'introduction de l'enseignement par activité dans une classe pour enseigner la physique est susceptible de répondre aux conditions précédentes, nouveauté et récurrence dans une temporalité réduite ; en effet, cet enseignement suppose que les élèves travaillent d'abord en autonomie sur une série de tâches successives en relation avec le savoir à s'approprier, celuici donnant la cohérence d'ensemble de la séquence d'enseignement correspondante. Le travail en autonomie sert de base à un travail collectif de formalisation mené dans un second temps (Vince et $\mathcal{E}$ al., 2016).

$\mathrm{Au}$ regard de cet ensemble d'éléments, nous souhaitons donc expliciter le contrat qui s'établit sur une séquence, lors de la mise en place d'un enseignement par activités dans une classe de physique, en nous centrant sur sa dimension « durable ». Nous souhaitons à la fois caractériser cette dimension du contrat en fin de séquence ainsi que la manière dont elle a évolué au cours de celle-ci. Pour cela, nous avons construit une méthode d'analyse que nous explicitons maintenant.

\section{MÉTHODOLOGIE}

Après avoir décrit sommairement le contexte de l'étude et évoqué le contenu thématique de la séquence observée, nous précisons la manière dont les données (principalement vidéo) ont été recueillies, le premier traitement qu'elles ont subi condui- 
Patrice Venturini \& Andrée Tiberghien

sant à la structuration de la séquence, et la manière dont elles ont été analysées à différentes échelles de temps. Enfin, la schématisation synthétique de l'ensemble des étapes du processus conclut cette section. Ces étapes s'organisent en quatre grandes phases, présentées dans la Figure 1 ci-dessous, que nous reprendrons tant dans l'exposé de la méthode que dans celui du résultat pour aider le lecteur à se situer en leur sein. La figure est présentée ici sans commentaire, elle prendra du sens dans les pages qui suivent.

Figure 1 : les quatre phases de la méthode d'investigation utilisée pour étudier la séquence d'enseignement qui comporte 4 activités

Ph 1 : Recueillir, transcrire et structurer les données

Ph 2 : Analyser l'activité 4 aux échelles $\mathrm{N}-2$ à $\mathrm{N}+1$, formaliser les règles correspondantes du contrat

Ph 3 : Analyser les activités 1,2 et 3 aux échelles $\mathrm{N}-2$ à $\mathrm{N}+1$, vérifier et compléter les règles établies sur l'activité 4

Ph 4 : Analyser la séquence aux échelles $\mathrm{N}+2$ et $\mathrm{N}+3$, formaliser les règles correspondantes du contrat

\section{Contexte de l'étude}

Le lycée où se déroule l'enseignement étudié se situe dans une zone à une trentaine de kilomètres d'une grande agglomération. Il recrute des élèves dans une zone périurbaine et plusieurs communes rurales, et comporte 1200 élèves. La classe observée est considérée par le professeur comme plutôt bonne mais hétérogène. La séquence observée se déroule au mois de novembre. Elle a été mise au point par un groupe de 12 enseignants dont l'enseignant de la classe observée et deux chercheurs dans le cadre du projet européen Assist-me ${ }^{2}$. Le professeur, qui travaillait pour la première fois dans un groupe de développement regroupant enseignants et chercheurs, a donc participé à son élaboration et les données ont été récoltées la première fois qu'il a enseigné la séquence conçue. À cette occasion, il a dû contribuer à l'installation d'un nouveau contrat dans la classe, une situation dans laquelle des ruptures par rapport au contrat établi en début d'année pouvaient apparaître qui étaient susceptibles de nous faciliter l'identification de nouveaux éléments du contrat. Ce choix a aussi déterminé la séquence observée.

\section{La séquence observée}

La séquence observée, fondée essentiellement sur un enseignement par activités, porte sur les phénomènes périodiques et leurs caractéristiques (période, cycle, fréquence) ; elle est dispensée en classe de seconde ( $10^{\mathrm{e}}$ année d'enseignement). Elle comporte quatre séances étalées sur deux semaines, à raison d'une heure en classe entière et une heure trente en demi-classe par semaine, soit cinq heures en tout. Le contenu lié à chacune des activités est précisé Figure 2. À chaque activité sont associés des documents (au moins l'énoncé de la tâche à réaliser par les élèves ${ }^{3}$ et un texte définissant les nouveaux concepts et/ou leurs relations) et éventuellement du matériel à disposition. Le format d'enseignement choisi par les concepteurs prévoit systématiquement un travail de groupe sur la tâche à réaliser, suivi d'une mise en commun et d'une institutionnalisation en classe entière (ou en demi-classe entière lorsque la séance est en demi-groupe ${ }^{4}$ ), tenant lieu de « correction » de la tâche. Ces deux phases forcément associées à une phase de présentation constituent pour nous "une activité ». Chaque activité est identifiée par son numéro lié à la chronologie de son introduction dans la classe.

Figure 2 : contenu des quatre activités constituant la séquence

Reconnaitre un phénomène périodique, son motif et sa période (Activité 1 Séance 1 en classe entière)

Définir la fréquence et sa relation à la période (Activité 2 Séance 1 en classe entière séance 2 en demi-classe)

Etudier un cas particulier de phénomène périodique, un haut-parleur (Activité 3 Séances 2 et 4 en demi-classe)

Etudier un autre cas particulier : les électrocardiogrammes (Activité 4 séance 4 en demi-classe)

La séance 3 non mentionnée ici comporte des tâches complémentaires venant en appui des activités 3 et 4 . Son contenu est ponctuellement évoqué dans les analyses. L'activité 4 est analysée de manière détaillée dans cet article. L'énoncé de la tâche à réaliser par les élèves et leur professeur durant cette activité est proposé dans l'annexe 1 p. 106. On y voit que l'objectif est de déterminer graphiquement la période des signaux correspondant à deux électrocardiogrammes (ECG) et de trouver ensuite la 
fréquence correspondante des battements cardiaques. Une série de questions aide les élèves à atteindre l'objectif assigné à l'activité : quel phénomène est traduit sur l'ECG; quelles grandeurs sont représentées sur l'ECG; identifier le motif du signal périodique ; identifier la grandeur (fréquence ou période) que l'on peut lire directement sur l'ECG; identifier l'ECG correspondant à un patient au repos ; mesurer la période des battements cardiaques d'un patient ; en déduire sa fréquence cardiaque. Ces questions sont traitées successivement en groupes de deux, puis en classe entière lors de la correction.

Nous présentons maintenant la première phase de la méthode utilisée qui comporte le recueil de données relatives à cette séquence, leur transcription puis leur structuration.

Figure 3: Phase 1 de la méthode, décrite dans les sections suivantes

Ph 1 : Recueillir, transcrire et structurer les données

Ph 2 : Analyser l'activité 4 aux échelles $\mathrm{N}-2$ à $\mathrm{N}+1$, formaliser les règles correspondantes du contrat

$\mathrm{Ph} 3$ : Analyser les activités 1,2 et 3 aux échelles $\mathrm{N}-2$ à $\mathrm{N}+1$, vérifier et compléter les règles établies sur l'activité 4

Ph 4 : Analyser la séquence aux échelles $\mathrm{N}+2$ et $\mathrm{N}+3$, formaliser les règles correspondantes du contrat

\section{Recueil des données}

Dans cet article, les données utilisées principalement sont un enregistrement vidéo de quatre séances avec une caméra centrée sur le professeur qui porte un microphone cravate HF. Les vidéos sont en effet particulièrement adaptées à une approche pragmatique dans laquelle s'inscrivent les outils théoriques utilisés (Tiberghien et Venturini, 2018). Les vidéos ont été ensuite transcrites en prenant en compte les propos des différents locuteurs ainsi que certains de leurs gestes. La transcription, complétée par des repères temporels fréquents, a été réalisée à l'aide du logiciel Transana. Dans les extraits que nous présentons, nous avons mis la ponctuation lorsque celle-ci facilitait la compréhension. Les documents distribués aux élèves ont également été récupérés.

\section{Structuration des données}

Chaque séance est structurée d'abord à l'échelle mésoscopique (10 à 30 minutes, niveau $\mathrm{N}$ ) en jeux didactiques. En lien avec la TACD et ses concepts, nous interprétons l'action didactique pour identifier les transactions successives. Nous repérons pour cela les évolutions importantes dans l'une ou plusieurs de ses trois dimensions, topo, chrono et mésogenétique, c'est-à-dire un changement significatif dans l'enjeu de savoir et/ou de la manière dont il est traité. Ces éléments, exprimés principalement du point de vue de l'action de l'élève, ont servi de base à la dénomination des jeux, par exemple «En collaborant avec son partenaire, exploiter deux ECG pour identifier et déterminer ses grandeurs caractéristiques ».

Nous structurons ensuite au sein de chaque jeu des épisodes de niveau N-1 à l'échelle de 1 à 5 minutes, épisodes identifiés comme étant les plus petites unités transactionnelles disposant d'une (relative) autonomie constitutives de la transaction. À ce titre, ils sont identifiés en recherchant les indices témoignant de modifications locales d'une ou plusieurs des dimensions de l'action didactique. Dans un souci opérationnel, leur titre reprend pour l'essentiel l'objet des échanges.

Enfin, nous avons souhaité analyser en détail la dernière activité ( $\left.\mathrm{n}^{\circ} 4\right)$ car nous avons supposé qu'à la fin de cette première séquence d'enseignement par le professeur, le contrat dans sa composante « durable » serait plus stabilisé qu'au cours des précédentes. Pour cette activité, nous identifions chaque acte verbal en complétant éventuellement pas des actes non verbaux qui nous ont paru significatifs au regard de l'avancée du savoir, à l'échelle microscopique de quelques secondes (niveau N-2).

Ces différentes délimitations, qui correspondent à des échelles déjà connues ne sont totalement stabilisées qu'en fin d'analyse, une fois l'action didactique totalement interprétée.

Ce premier traitement étant opéré et faisant apparaître des épisodes aux temporalités différentes, nous décrivons maintenant leurs analyses. Dans toutes les opérations de traitement que nous décrivons ci-dessous en allant de manière linéaire du niveau microscopique au niveau macroscopique, les caractéristiques d'un niveau temporel sont interprétées en relation avec les caractéristiques des niveaux supérieurs et inférieurs. En effet, selon Lemke (2000, p. 277) dont nous reprenons l'idée, pour comprendre ce qu'il se passe à une échelle de temps particulière, il faut au moins tenir compte des processus se dérou- 
lant aux niveaux immédiatement supérieur et immédiatement inférieur. Par ailleurs, le sens des signes identifiés à une échelle donnée est construit à des échelles supérieures puisque celles-ci donnent à chaque fois le contexte sans lequel les signes des échelles inférieures n'ont pas de sens (Lemke 2000).

La seconde phase de la méthode comporte plusieurs étapes que nous formalisons maintenant. Elle est, relative à l'analyse de l'activité 4 de l'échelle microscopique à celle de toute l'activité, et à la formalisation des règles correspondantes du contrat.

Figure 4 : Phase 2 de la méthode, décrite dans la section suivante

Ph 1 : Recueillir, transcrire et structurer les données

Ph 2 : Analyser l'activité 4 aux échelles $\mathrm{N}-2$ à $\mathrm{N}+1$, formaliser les règles correspondantes du contrat

Ph 3 : Analyser les activités 1,2 et 3 aux échelles $\mathrm{N}-2$ à $\mathrm{N}+1$, vérifier et compléter les règles établies sur l'activité 4

Ph 4 : Analyser la séquence aux échelles $\mathrm{N}+2$ et $\mathrm{N}+3$, formaliser les règles correspondantes du contrat

Traitement des données liées à l'activité 4 du niveau N-2 au niveau N+1 (échelle d'lh à 1 h 30)

Pour tous les jeux de l'activité 4, nous avons caractérisé chacun des actes constituant l'action didactique conjointe à l'aide des différents descripteurs, relatifs aux trois dimensions pragmatique, interactionnelle et représentationnelle. Cette analyse a été réalisée au sein d'un tableau Excel associé à chaque jeu, comportant chaque tour de parole, les descripteurs utilisés et leurs catégories. Le Tableau 2 illustre la manière dont nous avons procédé.

Prenons par exemple dans ce tableau, le tour de parole 3, émis par le professeur « ce n'est pas le motif élémentaire, c'est la? ", à la suite d'une proposition de l'élève selon laquelle la période d'un phénomène périodique est un motif élémentaire. Ce tour de parole comporte deux actes discursifs du professeur, «ce n'est pas le motif élémentaire » et " c'est la?». «Ce n'est pas le motif élémentaire » est un acte qui d'un point de vue pragmatique relève d'une évaluation, et sur le plan interactionnel d'une poursuite évaluative. "C'est la? » est un acte qui d'un point de vue pragmatique relève d'une demande de proposition, et d'une question sur le plan interactionnel. Aucun des deux actes ne peut être analysé du point de vue représentationnel puisque aucun ne délivre une information sur la période.

Le tour de parole 4 comporte un seul acte discursif émis par les élèves : « la durée ». Sur le plan pragmatique, il s'agit d'une proposition effectuée par l'élève lui-même, identifiée comme une réponse sur le plan interactionnel. Sur le plan représentationnel, la facette correspondante, reprise par le professeur au tour de parole 5 « la période est la durée du motif élémentaire » correspond à une mise en relation entre « observable » (le motif sur le graphe) et modèle (définition de période); elle est exprimée en langue scientifique et relève de la catégorie de facettes " période » qui rassemble toutes les facettes en lien avec ce concept. Enfin, sur le plan de la tâche épistémique mise en jeu, elle correspond à une interprétation.

Nous avons ensuite récapitulé ces analyses au sein de chacun des épisodes N-1 en comptabilisant chacune des modalités des différents descripteurs. Nous avons recherché des régularités, des caractéristiques communes à différents épisodes N-1 de chacun des jeux, dans le but de les interpréter comme appartenant à la dimension durable du contrat. Nous avons pu ainsi observer à cette échelle des tendances identiques pour la plupart des épisodes N-1 appartenant à un même jeu ou en d'autres termes leur cohérence (cf. résultats). Cela nous a permis de récapituler les caractéristiques des actes au niveau de chacun des jeux à l'échelle mésoscopique $(\mathrm{N})$, de décrire ainsi l'action didactique à cette échelle et de l'interpréter en termes de contrat sous forme de règles.

L'action didactique ainsi décrite au niveau mésoscopique de chacun des jeux et résumée par leur intitulé a ensuite été examinée à l'échelle de $1 \mathrm{~h}$ à $1 \mathrm{~h} 30$ qui est celle de l'activité. L'action didactique est alors apparue structurée à cette échelle de niveau N+1 (cf. résultats correspondants). Cette structure qui constitue une interprétation des caractéristiques de chacun des jeux à cette échelle, a alors été associée à de nouvelles règles constitutives du contrat.

Dans la phase suivante de la méthode, il s'agit de tester la validité des règles inférées à partir de l'analyse de l'activité 4 sur les autres activités de la séquence et éventuellement de les compléter. 
Tableau 2 : Extrait du tableau de caractérisation des actes selon les dimensions pragmatique, interactionnelle et représentationnelle

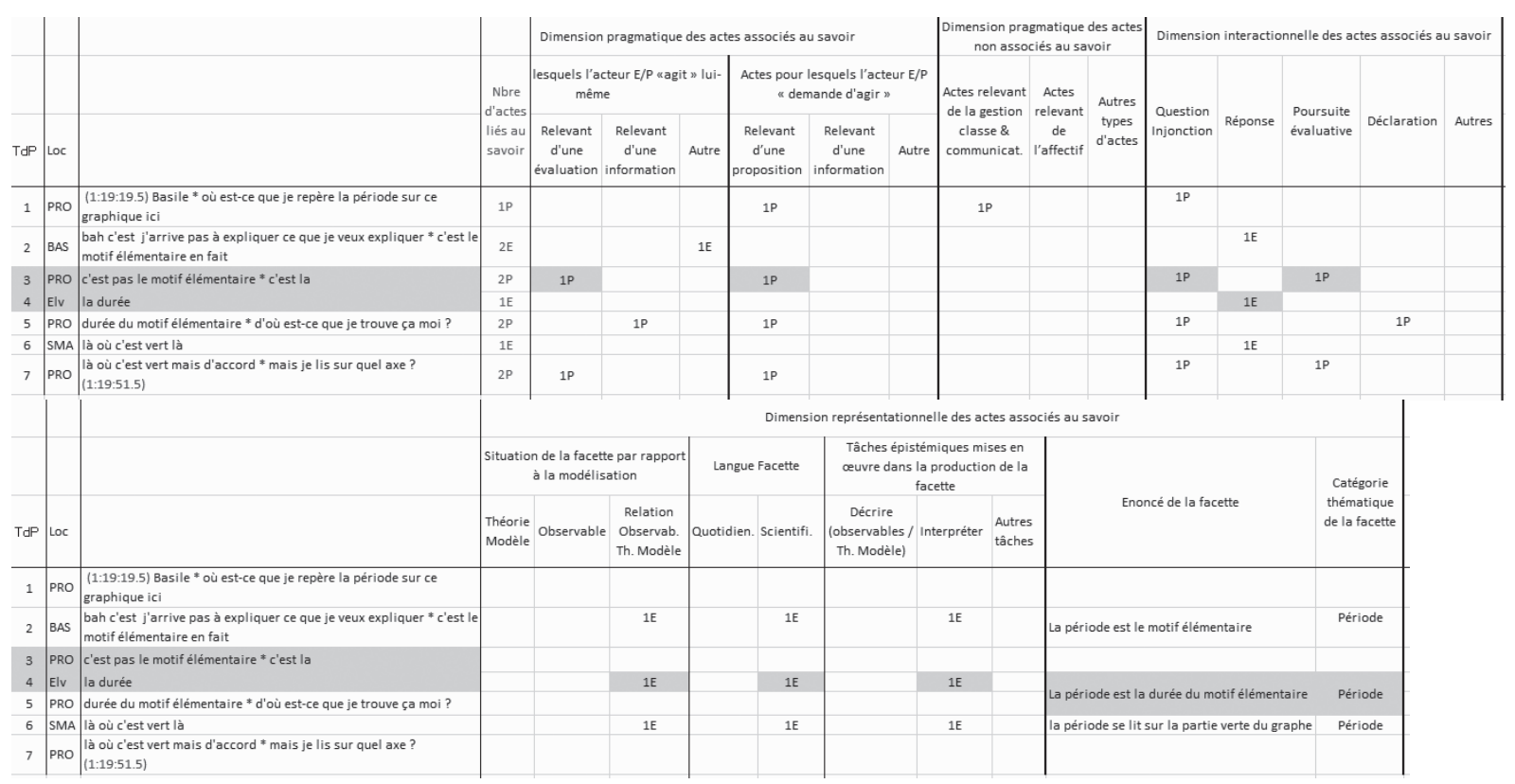

L'extrait de la transcription est issu de la correction en classe entière de l'activité 4 (séance 4), le graphique est projeté sur un écran par le professeur ( $1 \mathrm{P}=1$ acte du professeur ; $2 \mathrm{E}=2$ actes de l'élève).

Figure 5 : Phase 3 de la méthode, décrite dans la section suivante

\section{Ph 1 : Recueillir, transcrire et structurer les données}

Ph 2 : Analyser l'activité 4 aux échelles $\mathrm{N}-2$ à $\mathrm{N}+1$, formaliser les règles correspondantes du contrat

Ph 3 : Analyser les activités 1,2 et 3 aux échelles $\mathrm{N}-2$ à $\mathrm{N}+1$, vérifier et compléter les règles établies sur l'activité 4

Ph 4 : Analyser la séquence aux échelles $\mathrm{N}+2$ et $\mathrm{N}+3$, formaliser les règles correspondantes du contrat

\section{Traitement des données liées aux trois autres activités du niveau $\mathrm{N}-2$ au niveau $\mathrm{N}+1$}

L'analyse du déroulement de l'activité 4 nous permet de préciser certaines composantes du contrat didactique aux niveaux $\mathrm{N}$ et $\mathrm{N}+1$. Il reste à voir si celles-ci ont évolué ou au contraire sont stables au cours des trois premières activités et si des composantes sont restées inaperçues dans les analyses de l'activité 4. En effet, dans le cas où elles auraient été établies précédemment et où les actes du professeur et des élèves auraient été en adéquation avec elles dans l'activité 4 , les comportements associés ne feraient pas l'objet d'une explicitation au cours de cette activité.

Il était difficile de renouveler le type d'analyse réalisée pour l'activité 4 en raison du temps que cela demande. Aussi, nous avons procédé différemment et avons choisi de mener deux analyses. La première vise à vérifier la validité des règles les plus importantes du contrat en mobilisant comme dans les analyses précédentes la quantification relative aux différentes modalités des actes. Pour cela, nous évaluons dans chaque épisode N-1 si les actes dont les caractéristiques sont associées à chacune des règles les plus importantes, sont majoritaires. La seconde est menée avec une approche thématique du discours de la classe, chaque règle établie pour l'activité 4 constituant un "thème ». Nous relisons alors les transcriptions (avec si nécessaire les vidéos en appui) en sélectionnant les extraits relatifs à ce thème. Les extraits peuvent ou non confirmer la règle correspondante.

Enfin, la dernière phase de la méthode est relative à l'analyse de la séquence à des échelles de temps supérieures $(\mathrm{N}+2, \mathrm{~N}+3)$ à celle de l'activité $(\mathrm{N}+1)$ et à la formalisation des règles correspondantes du contrat. 
Figure 6: Phase 4 de la méthode, décrite dans la section suivante

\section{Ph 1 : Recueillir, transcrire et structurer les données}

Ph 2 : Analyser l'activité 4 aux échelles $\mathrm{N}-2$ à $\mathrm{N}+1$ formaliser les règles correspondantes du contrat

Ph 3 : Analyser les activités 1, 2 et 3 aux échelles $\mathrm{N}-2$ $\mathrm{N}+1$, vérifier et compléter les règles établies sur l'activité 4

Ph 4 : Analyser la séquence aux échelles $\mathrm{N}+2$ et $\mathrm{N}+3$, formaliser les règles correspondantes du contrat

Traitement des données relatives à l'ensemble de la séquence aux échelles supérieures

Disposant des règles liées à la dimension durable du contrat jusqu'au niveau $\mathrm{N}+1$ pour la totalité de la séquence, nous avons alors interprété les analyses effectuées au niveau N+1 à l'échelle de 2 à 3 h. C'est en effet à cette échelle (niveau N+2) que l'on voit apparaître une autre structure de l'action didactique à laquelle de nouvelles règles sont associées (cf. résultats correspondants). De la même manière, en interprétant les caractéristiques du niveau $\mathrm{N}+2$ à l'échelle macroscopique de la séquence $(\mathrm{N}+3)$, nous avons dégagé la dernière composante du contrat.

Schématisation synthétique de la méthodologie

Nous proposons une synthèse de la méthode exposée dans les paragraphes précédents en termes d'échelles temporelles (Figure 7) ainsi qu'une synthèse d'un point de vue chronologique détaillant ses quatre phases (Figure 8).

Figure 7 : Schéma synthétique de la méthode selon les échelles concernées

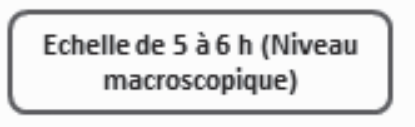

Echelle de $2 \mathrm{~h} 30$ à $3 \mathrm{~h}$

Echelle de $1 \mathrm{~h}$ à $1 \mathrm{~h} 30$

Echelle de 10 à $\mathbf{3 0}$ minutes (Niveau Mésoscopique)

Echelle de 1 à 5 min

Echelle de quelques secondes (Niveau microscopique)

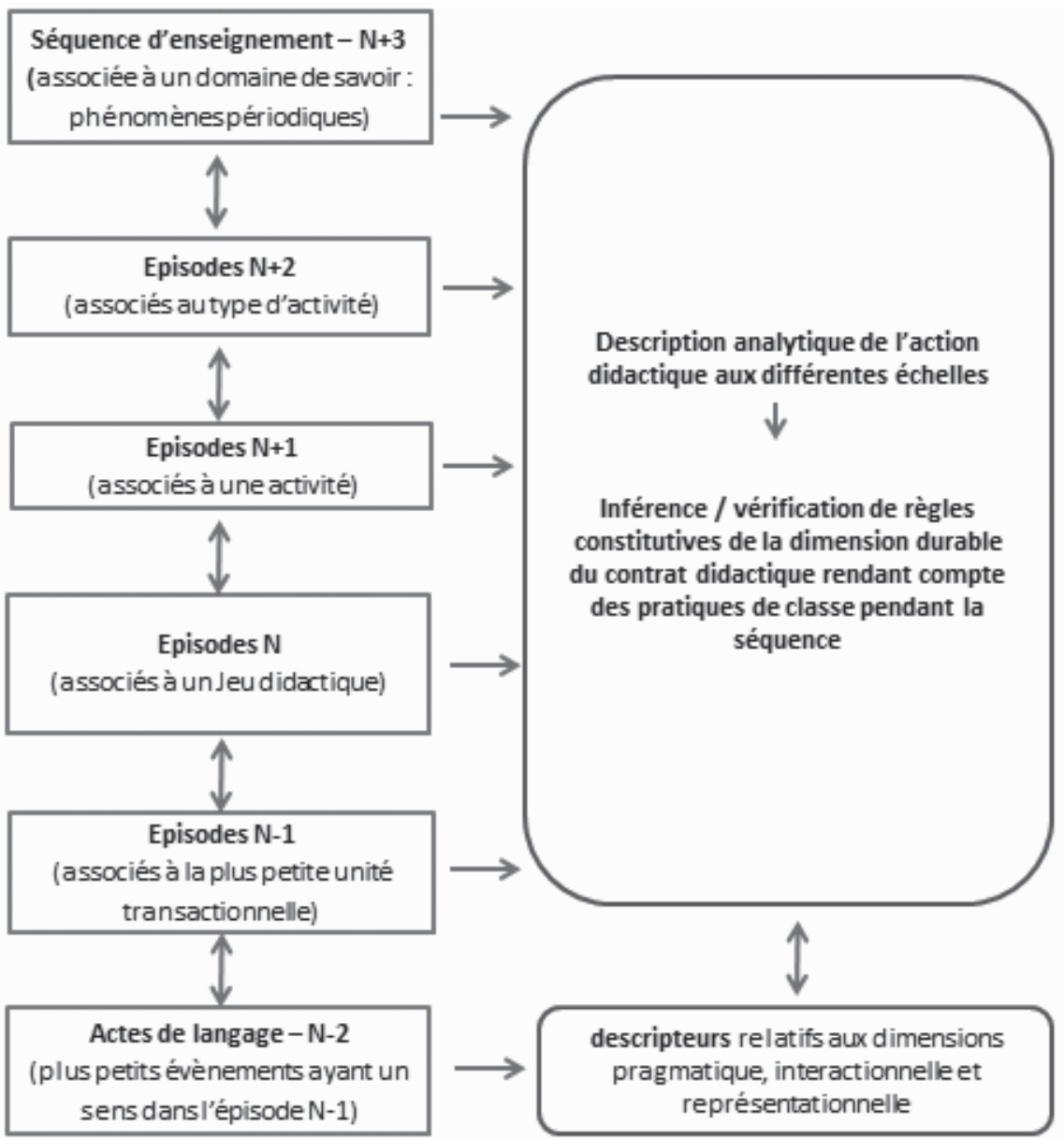


Figure 8 : Schéma chronologique de la méthodologie de détermination du contrat didactique sur une séquence d'enseignement par activités

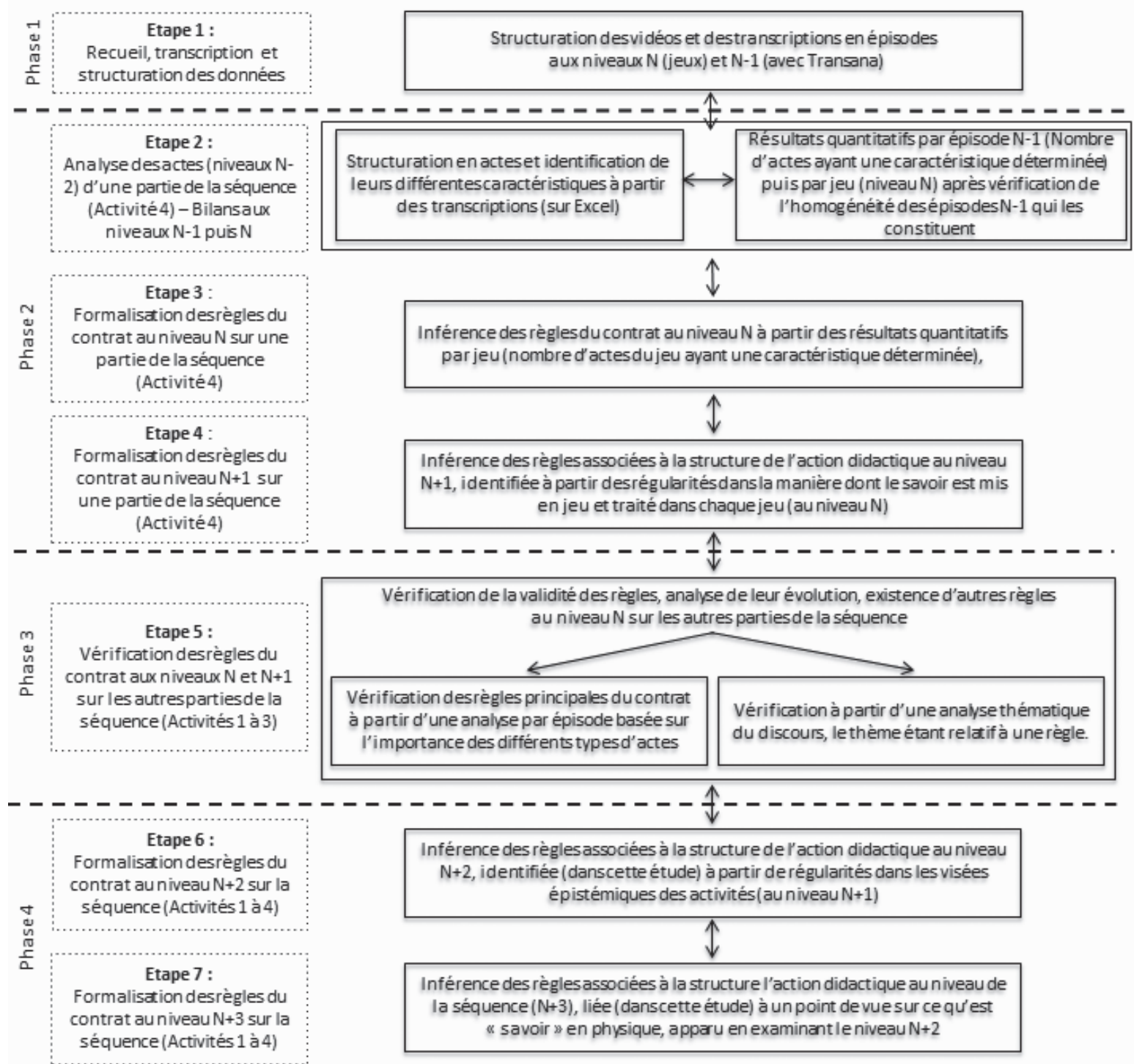

Les éléments sur lesquels la structure de l'action didactique est basée (dans cette étude) aux niveaux N+1, N+2 et N+3, sont donnés sans commentaires dans le schéma, leur justification est à trouver dans les résultats présentés plus loin.

Nous présentons maintenant les résultats, développés en trois étapes. Tout d'abord, nous rapportons les analyses des niveaux N-2 à N qui nous amènent à construire des composantes du contrat lors du déroulement de l'activité 4, d'abord à l'échelle mésoscopique (Niveau N) puis à l'échelle de la durée de l'activité (1h - 1 h 30, Niveau N+1). Nous analysons ensuite dans quelle mesure les règles établies pour l'activité 4 valent également pour les autres activités ou si elles évoluent au cours du temps. Nous fournissons enfin les composantes du contrat issues des analyses aux échelles de temps supérieures $\mathrm{N}+2$ (étape 6) et $\mathrm{N}+3$ (étape 7). 


\section{RECONSTRUCTION DU CONTRAT LIÉ AU DÉROULEMENT DE L'ACTIVITÉ 4}

En nous fondant sur l'analyse des actes du professeur et des élèves, nous commençons par donner d'abord les composantes de ce contrat à l'échelle mésoscopique $\mathrm{N}$ avant d'aborder dans la seconde partie les composantes établies à l'échelle $\mathrm{N}+1$. Ces analyses, qui mettent en jeu les échelles $\mathrm{N}-2, \mathrm{~N}-1 \mathrm{~N}$ et $\mathrm{N}+1$ correspondent à la seconde phase de la méthode d'investigation décrite précédemment (étapes 2, 3 et 4 dans la Figure 8 ) et permettent d'inférer des règles du contrat pour l'activité 4 .

Figure 9 : les sections suivantes décrivent les résultats issus de la phase 2 de la méthode d'investigation

Ph 1 : Recueillir, transcrire et structurer les données

Ph 2 : Analyser l'activité 4 aux échelles $\mathrm{N}-2$ à $\mathrm{N}+1$, formaliser les règles correspondantes du contrat

Ph 3 : Analyser les activités 1,2 et 3 aux échelles $\mathrm{N}-2$ à $\mathrm{N}+1$, vérifier et compléter les règles établies sur l'activité 4

Ph 4 : Analyser la séquence aux échelles $\mathrm{N}+2$ et $\mathrm{N}+3$,

formaliser les règles correspondantes du contrat

Contrat lié au déroulement de l'activité 4 établi à l'échelle mésoscopique $\mathrm{N}$
Nous décrivons ici les résultats obtenus dans les étapes 2 et 3 apparaissant dans la Figure 8.

Après avoir donné une vue d'ensemble des niveaux $\mathrm{N}, \mathrm{N}-1$ et $\mathrm{N}-2$, nous rapportons l'analyse systématique des différentes dimensions pragmatique, interactionnelle et représentationnelle des actes du professeur et des élèves. Celle-ci, effectuée au niveau microscopique N-2, permet d'aborder l'importance et la forme des contributions du professeur et des élèves dans le savoir en jeu dans la classe. En considérant que la très grande majorité des épisodes N-1 d'un même jeu ont des caractéristiques communes (ce que nous montrerons), nous avons récapitulé les analyses microscopiques directement au niveau mésoscopique de chacun des jeux (niveau $\mathrm{N}$ ) pour les présenter, et les exploiter ensuite pour décrire les particularités de l'action didactique dans chacun des jeux, et enfin d'interpréter cet ensemble en termes de contrat.

\section{Vue d'ensemble aux niveaux $\mathrm{N}, \mathrm{N}-1$ et $\mathrm{N}-2$ structurant le déroulement de l'activité 4}

L'activité 4, menée au sein de la demi-classe (18 élèves), est structurée en épisodes de niveau $\mathrm{N}$ (jeux) et $\mathrm{N}-1^{5}$ dont le Tableau 3 récapitule les caractéristiques générales. Rappelons que le découpage des épisodes de niveau $\mathrm{N}$ est obtenu en recherchant

Tableau 3 : Caractéristiques générales des épisodes de niveaux N (jeux) et

$\mathrm{N}-1$ résultant de la structuration du déroulement de l'activité 4

\begin{tabular}{|c|c|c|c|c|c|c|}
\hline & & \multicolumn{3}{|c|}{ Nombre d'épisodes de niveau N-1 } & \multirow{2}{*}{$\begin{array}{l}\text { Durée } \\
\text { moyenne } \\
\text { des } \\
\text { épisodes }\end{array}$} & \multirow{2}{*}{$\begin{array}{l}\text { Nombre } \\
\text { total } \\
\text { d'actes }\end{array}$} \\
\hline $\begin{array}{l}\text { Jeu / durée (min: } \\
\text { sec)/ } \\
\text { Organisation de la } \\
\text { classe }\end{array}$ & Intitulé & Total & $\begin{array}{l}\text { Centrés sur } \\
\text { le savoir }\end{array}$ & $\begin{array}{l}\text { Centrés sur } \\
\text { la gestion } \\
\text { classe }\end{array}$ & & \\
\hline $\begin{array}{l}\text { S4 J8 } \\
03: 32 \\
\text { Classe entière }\end{array}$ & $\begin{array}{l}\text { Prendre connaissance de l'activité propo- } \\
\text { sée visant à exploiter deux électrocardio- } \\
\text { grammes pour identifier et déterminer leurs } \\
\text { grandeurs caractéristiques }\end{array}$ & 1 & 1 & 0 & & 5 \\
\hline $\begin{array}{l}\text { S4 J9 } \\
22: 23 \\
\text { Petits groupes }\end{array}$ & $\begin{array}{l}\text { En collaborant avec son partenaire, exploi- } \\
\text { ter deux ECG pour identifier et déterminer } \\
\text { ses grandeurs caractéristiques (fréquence } \\
\text { cardiaque période) }\end{array}$ & 23 & $\begin{array}{l}17 \\
\text { (Durée } \\
\text { totale } \\
19: 45)\end{array}$ & $\begin{array}{l}6 \\
\text { (Durée } \\
\text { totale } \\
2: 38)\end{array}$ & $1: 19$ & 261 \\
\hline $\begin{array}{l}\text { S4 J10 } \\
14: 47 \\
\text { Classe entière }\end{array}$ & $\begin{array}{l}\text { Comprendre grâce à la discussion collec- } \\
\text { tive la correction de la détermination de la } \\
\text { fréquence et la période de deux ECG }\end{array}$ & 7 & 7 & 0 & $2: 07$ & 236 \\
\hline
\end{tabular}

La dénomination des jeux inclut les numéros de séance et du jeu dans la séance, par exemple S4 J87 ). Les épisodes sont déclarés "centrés sur le savoir " lorsque les actes relatifs au savoir qu’ils comportent sont prédominants par rapport aux actes relatifs à la gestion de la classe, et « centrés sur la gestion de la classe » dans le cas inverse. 
les indices témoignant de modifications d'une ou plusieurs des dimensions de l'action didactique, et celles des épisodes de niveau N-1 en recherchant des indices de modifications locales de ces mêmes dimensions. Ces dernières sont particulières dans le jeu S4 J9 au cours duquel les élèves travaillent en petits groupes de deux : le changement d'épisode $\mathrm{N}-1$ est déterminé par l'action du professeur qui va d'un groupe à un autre, ou alors s'adresse à la classe entière $^{6}$. Nos données ne donnent pas d'information directe sur ce que font les petits groupes en dehors des moments d'intervention du professeur. On trouve dans les annexes 2 p. 105 et 3 p. 106 le Tableau 18 et le Tableau 19 présentant respectivement les épisodes $\mathrm{N}-1$ des jeux S4 J9 et S4 J10.

Le Tableau 3 montre que l'activité 4 d'une durée d'un peu plus de 40 minutes est structurée en trois jeux, le premier très court, le deuxième, le plus long, contient 23 épisodes $\mathrm{N}-1$ et le dernier un peu plus courts en contient 7 . Il montre aussi qu'une minorité d'épisodes N-1 (6 sur 31) est dédiée à la gestion de la classe. Ces épisodes sont tous situés dans le jeu S4 J9 et leur durée totale est très faible (2:38) au regard de la durée totale de l'activité (40:42). L'essentiel du déroulement de l'activité 4 est donc consacré à des échanges centrés sur les savoirs, après un jeu S4 J8 où les interactions sont réduites au minimum (5 actes en 3:32), les élèves prenant connaissance de la tâche à réaliser.

Dans le jeu S4 J9, les épisodes N-1 ont pour la plupart une durée courte (1:19 de moyenne, 10 épisodes ont une durée inférieure à 1:00); la très grande majorité des épisodes centrés sur le savoir (13 sur 17) sont relatifs au phénomène périodique et à ses caractéristiques, le savoir enjeu de l'activité. Le réseau conceptuel associe le cycle, la période, la fréquence ${ }^{8}$. Le Tableau 18 (cf. annexe 2) montre que, au début du jeu, seulement deux épisodes sont consacrés à la tension qui apparaît dans l'électrocardiogramme et deux autres à la signification des termes « repérer » et " grandeur » qui figurent dans l'énoncé, si bien qu'après les questions traitées dans ces quatre groupes ( 6 premières minutes de l'activité), on peut considérer que la majorité des élèves a compris l'énoncé de la tâche et traite les questions posées. Par ailleurs, compte tenu du nombre de groupes (9) et de la durée dédiée au savoir dans le jeu (19:45), on peut affirmer que l'essentiel du travail des élèves se déroule au cours du jeu S4 J9 en l'absence du professeur, celui-ci passant en moyenne juste un peu plus de 2 minutes dans chaque groupe.

Dans le jeu S4 J10 correspondant à la correction en classe entière de l'activité, les sept épisodes correspondent aux sept questions de l'énoncé de la tâche (voir les questions dans l'annexe 1 et le découpage en épisodes N-1 Tableau 19, annexe 3). Ainsi le professeur assure une correction très fidèle à l'énoncé. Ces épisodes sont homogènes du point de vue du savoir, celui-ci se limitant au même réseau conceptuel mobilisé dans le jeu S4 J9, et du point de vue de la durée (6 épisodes font moins de 3 minutes).

Ainsi, les épisodes appartenant à chacun des jeux S4 J9 et S4 J10 sont homogènes, à la fois pour ce qui est de leur durée et de leur centration sur l'enjeu du savoir.

\section{Analyse des actes (niveau N-2)}

Après avoir exploré les caractéristiques générales des jeux (niveau N) et épisodes de niveau N-1 issus de la structuration de la séquence, nous donnons maintenant les résultats de l'analyse des actes du professeur et des élèves, associés au découpage microscopique en actes (niveau N-2).

\section{Dimension pragmatique}

Vue d'ensemble

Tableau 4: Vue d'ensemble des actes de professeur et des élèves pour les jeux de l'activité 4

\begin{tabular}{|l|c|c|c|}
\hline Jeu & S4 J8 & S4 J9 & S4 J10 \\
\hline Total des actes du jeu & 5 & 261 & 236 \\
\hline $\begin{array}{l}\text { Total des actes relatifs au } \\
\text { savoir }\end{array}$ & 4 & 233 & 234 \\
\hline $\begin{array}{l}\text { Actes du Professeur rela- } \\
\text { tifs au savoir }\end{array}$ & 2 & 133 & 156 \\
\hline $\begin{array}{l}\text { Actes des Élèves relatifs } \\
\text { au savoir }\end{array}$ & 2 & 100 & 78 \\
\hline $\begin{array}{l}\text { \% d'actes de savoir sur le } \\
\text { total des actes }\end{array}$ & $89 \%$ & $99 \%$ \\
\hline $\begin{array}{l}\text { Parmi les actes relatifs au } \\
\text { savoir, } \\
\% \text { d'actes du Professeur } \\
\% \text { d'actes des Élèves }\end{array}$ & $57 \%$ & $67 \%$ \\
\hline $\begin{array}{l}\text { Nombre d'actes relatifs au } \\
\text { savoir par minute }\end{array}$ & $10 \%$ & $16 \%$ \\
\hline
\end{tabular}

Les pourcentages ne sont pas donnés pour le jeu S4 J8 en raison de son faible nombre d'actes (Analyse au niveau N-2). 
Patrice Venturini \& Andrée Tiberghien

L'analyse avec un regard pragmatique de la nature des actes confirme celle conduisant à la structuration de l'action didactique aux niveaux $\mathrm{N}$ et $\mathrm{N}-1$ : les actes relatifs au savoir sont très largement prédominants dans les jeux S4 J8 et S4 J9 (4 sur 5 et $89 \%$ ) et quasiexclusifs dans le jeu S4 J10 (99\%). La différence dans les fréquences des actes relatifs au savoir entre les jeux S4 J9 et S4 J10 (10 actes/min et 16 actes/min) s'explique d'une part, par le fait que dans le jeu S4 $\mathrm{J} 9$, le professeur va d'un groupe à un autre et prend le temps de regarder leur production et d'autre part, dans le jeu S4 J10, il dirige la correction sans interruption et toutes ses interventions ainsi que celles des élèves concernent le savoir.

Examinons maintenant plus en détail les deux jeux principaux de l'activité 4 au regard des deux types d'actes relatifs au savoir que nous avons proposés dans la méthodologie selon que l'acteur concerné, professeur ou élève, « agit lui-même » ou « demande (à son interlocuteur) d'agir » sur le savoir.

Actes des types « agir » et " demande d'agir » (sur le savoir)

Le Tableau 5 met en évidence l'importante contribution des élèves dans le jeu S4 J9 (43\% des actes relatifs au savoir), même si elle est un peu inférieure à celle du professeur (57\%). Pour le jeu S4 J10, la contribution des élèves est moins importante mais leurs actes relatifs au savoir représentent encore la moitié de ceux du professeur et donc un tiers des actes de savoir. Cette tendance se vérifie dans une majorité d'épisodes $\mathrm{N}-1$ : dans 12 épisodes sur les 17 du jeu S4 J9, la contribution des élèves aux actes liés au savoir est supérieure à $40 \%$ et elle dépasse $30 \%$ dans 5 des 7 épisodes du jeu S4 J10.
Toutefois, il existe des différences entre les jeux S4 J9 et S4 J10.

Dans le jeu S4 J9, le professeur demande aux élèves d'agir sur le savoir plus souvent qu'il ne le fait lui-même ( $57 \%$ vs $43 \%$ des actes liés au savoir) tandis que les élèves agissent directement sur le savoir dans la quasi-totalité de leurs actes (94\%) (cf. Tableau 5) généralement en faisant des propositions (cf. Tableau 6). Ces deux constatations valent pour une très grande majorité d'épisodes N-1 (respectivement dans 12 et 14 épisodes sur 17). De plus, la différence entre les actes de type « $\mathrm{P}$ agit » et "P demande d'agir » dans chacun des deux jeux est confirmée statistiquement (hypothèse nulle rejetée : $\mathrm{p}<0.01$, chi $2=9,102$ pour degré de liberté $=1$ ). En revanche, le même test montre qu'il n'y a pas de différence dans le cas des élèves qui « agissent » dans les deux jeux.

L'Extrait 1 illustre des « demandes d'agir » fréquentes de l'enseignant (demande sur la manière dont la question a été comprise, demande de rappeler une définition après une proposition erronée du groupe que le professeur n'évalue pas) et les propositions correspondantes des élèves. De fait, les demandes d'agir du professeur aident les élèves à contribuer à l'avancée des savoirs. Les demandes de définition du professeur amenant l'élève à relier la période et le cycle permettent à celui-ci de réaliser que la période n'est pas associée à une seconde, mais à la durée d'un cycle.

Tableau 5: Répartition des actes relatifs au savoir entre le professeur et l'élève, et pour chacun d'entre eux, répartition entre acte lié au savoir et demande d'acte lié au savoir (Analyse au niveau N-2)

\begin{tabular}{|c|c|c|c|c|c|c|}
\hline & \multicolumn{2}{|c|}{$\begin{array}{c}\text { Répartition des actes } \\
\text { relatifs au savoir }\end{array}$} & \multicolumn{2}{c|}{$\begin{array}{c}\text { Actes du Professeur rela- } \\
\text { tifs au savoir }\end{array}$} & \multicolumn{2}{c|}{$\begin{array}{c}\text { Actes des Élèves } \\
\text { relatifs au savoir }\end{array}$} \\
\hline & Professeur & Élèves & P Agit & $\begin{array}{c}\text { P Demande } \\
\text { d'agir }\end{array}$ & $\begin{array}{c}\text { E Agit } \\
\text { E Demande } \\
\text { d'agir }\end{array}$ \\
\hline Jeu S4 J9 & $57 \%$ & $43 \%$ & $42 \%$ & $58 \%$ & $94 \%$ & $6 \%$ \\
N =233 & $(133)$ & $(100)$ & $(56)$ & $(77)$ & $(94)$ & $(6)$ \\
\hline Jeu S4 J10 & $67 \%$ & $33 \%$ & $60 \%(94)$ & $40 \%$ & $96 \%$ & $4 \%$ \\
N =234 & $(157)$ & $(77)$ & $(63)$ & $(74)$ & $(3)$ \\
\hline
\end{tabular}


Extrait 1

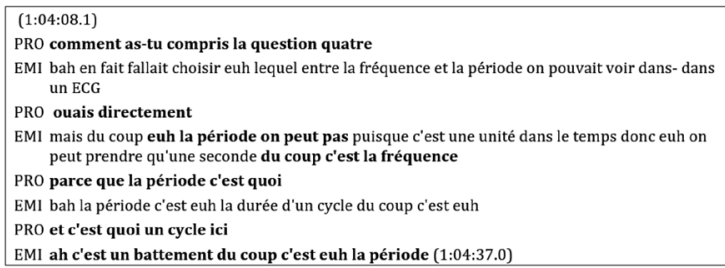

Le professeur demande à un groupe comment il a compris la question et valide sa réponse tout en la complétant. Cela conduit l'élève à faire une proposition de réponse, mais celle-ci est erronée. Le professeur poursuit alors en demandant la définition de la période, ce qui aide l'élève à formuler une réponse pertinente (S4 J9 Ep o) Dans les extraits, les éléments en gras viennent plus spécifiquement en appui de l'analyse, que celle-ci soit détaillée dans le texte de l'article, ou résumée dans la légende de l'extrait.

Extrait 2

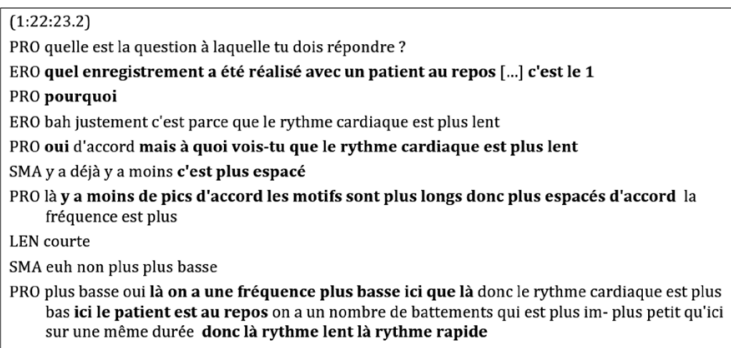

Le professeur demande aux élèves de faire des propositions en réponse à une des questions de la tâche. Les élèves proposent les résultats qu’ils ont préparés durant leur travail en groupe, qu'ils doivent justifier et qui contribuent à l'avancée du savoir. Le professeur valide et institutionnalise la réponse (S4 J10 Ep 5).
À l'inverse du jeu S4 J9, dans le jeu S4 J10 au cours duquel on corrige en classe entière la tâche, le professeur « agit » lui-même sur le savoir plus qu'il ne « demande d'agir » $(60 \%$ vs $40 \%$ de ses actes relatifs au savoir). En revanche comme dans le jeu S4 J9, l'élève agit dans la quasi-totalité de ses actes (96\%) généralement en faisant des propositions (cf. Tableau 6), fondées sur le travail réalisé en groupes et qui contribuent à l'avancée des savoirs, comme le montre l'Extrait 2. Ce comportement des acteurs se retrouve dans une grande majorité des sept épisodes N-1 de ce jeu : cinq pour le professeur et sept pour les élèves.

Les deux extraits précédents, tout comme le Tableau 6, montrent en quoi le comportement du professeur change entre les deux jeux quand il agit lui-même sur le savoir. Si la proportion des évaluations est équivalente, dans le jeu S4 J10, les propositions du professeur sont moindres, et les informations liées au savoir ou à la tâche sont plus nombreuses (comme dans la dernière intervention de $\mathrm{P}$ dans l'Extrait 2) : le professeur assume ici la responsabilité du savoir. D'ailleurs, la différence entre les modalités d'action du professeur lorsque celui-ci agit sur le savoir dans chacun des deux jeux est confirmée statistiquement (hypothèse nulle rejetée : $\mathrm{p}<0.01$, chi2 = 9.503, degré de liberté = 2).

Les extraits précédents montrent aussi que les propositions faites par les élèves, en petit groupe ou classe entière, contribuent à l'avancée du savoir, même si parfois c'est de manière minime. Par contre, dans les deux jeux analysés, on constate qu'ils demandent très peu au professeur « d'agir lui-même sur le savoir » (cf. Tableau 5), par exemple en lui demandant d'apporter des précisions. Cette possibilité semble toutefois rester ouverte dans la classe : on

Tableau 6 : Catégories d'actes majoritaires chez le professeur et l'élève lorsqu'ils « agissent » eux-mêmes sur le savoir (Analyse au niveau N-2)

\begin{tabular}{|c|c|c|c|c|}
\hline & \multicolumn{3}{|c|}{$\begin{array}{c}\text { Actes dans lesquels « le professeur agit sur le savoir » } \\
\text { Jeu S4 J9: } N=56 \\
\text { Jeu S4 J10:N }=94\end{array}$} & \multirow{2}{*}{$\begin{array}{c}\text { Actes dans lesquels } \mathrm{E} \text { « agit sur } \\
\text { le savoir » } \\
\text { Jeu S4 J9: } \mathrm{N}=94 \\
\text { Jeu S4 J10 : } \mathrm{N}=74 \\
\text { relevant d'une proposition }\end{array}$} \\
\hline & $\begin{array}{l}\text { relevant d'une } \\
\text { proposition }\end{array}$ & $\begin{array}{l}\text { relevant d'une } \\
\text { évaluation }\end{array}$ & $\begin{array}{l}\text { relevant d'une information sur le } \\
\text { savoir ou sur la tâche }\end{array}$ & \\
\hline Jeu 9 & $13-23 \%$ & $27-48 \%$ & $10-18 \%$ & $71-76 \%$ \\
\hline Jeu 10 & $8-9 \%$ & $43-46 \%$ & $34-36 \%$ & $70-94 \%$ \\
\hline
\end{tabular}


E sur 100 actes liés au savoir dans le jeu S4 J9; leur absence dans le jeu S4 J10 peut être interprétée en avançant l'idée que le travail réalisé en petits groupes a été effectif et permet aux élèves de comprendre le contenu des échanges lors de la correction. Quant au jeu S4 J9, dans 10 épisodes N-1 sur 17, le professeur commence par lire les productions des élèves quand il arrive dans le groupe et pose ensuite une question à partir de cette prise d'information. Ce comportement suppose d'une part qu'il décode alors les difficultés et les incertitudes des élèves et que les échanges qui suivent y répondent en partie, et d'autre part que les élèves écrivent tout ou partie de leur cheminement et de leur réponse. Dans l'activité 4, l'obligation de faire état du cheminement est implicite, jamais le professeur ne le demande mais son comportement en témoigne parfois. Ainsi, dans l'épisode i, min $57: 18$, le professeur passe six secondes à prendre connaissance du cahier des élèves après s'être plaint : «alors j'arrive pas à lire dans ce sens-là ».

\section{Dimension interactionnelle}

Un regard global sur le Tableau 7 résumant l'analyse interactionnelle confirme l'analyse pragmatique.

Les tests statistiques montrent que les actes du professeur sont différents selon les deux jeux sur le plan interactionnel (rejet de l'hypothèse nulle : $\mathrm{p}<0.001$ chi2 $=19.526, \mathrm{dl} 3$ ), alors qu'ils ne permettent pas de différencier ceux des élèves sur ces mêmes jeux.

D'une part, les élèves mettent en œuvre du savoir mais à l'initiative du professeur ; en effet, les questions et injonctions sont l'œuvre du professeur, et les réponses sont le fait des élèves, celles-ci constituant l'essentiel de leurs interventions (86 et $94 \%$ ). Le taux des réponses dans les actes des élèves reliés au savoir est d'ailleurs supérieur à $80 \%$ dans 11 épisodes sur 17 dans le jeu S4 J9 et dans 6 épisodes sur 7 dans le jeu S4 J10, et le « débit » des réponses est régu- lier tout au long des deux jeux, situé entre 0.5 et 1 réponse toutes les 10 secondes en moyenne dans chacun des épisodes $\mathrm{N}-1$.

D'autre part, le professeur assume la responsabilité des savoirs nettement plus dans le jeu S4 J10 que dans le jeu S4 J9. En effet, la tendance selon laquelle le professeur préférentiellement " demande d'agir » dans le jeu S4 J9 et « agit » sur le savoir » dans le jeu S4 J10 se retrouve logiquement ici dans l'évolution à la baisse du taux de ses questions et injonctions entre les deux jeux ( $62 \%$ vs $41 \%$ ). Cette évolution se fait au profit des taux de déclaration et de poursuite évaluative qui eux, augmentent. Elle peut être reliée à la nature du jeu S4 J10 dans lequel il s'agit de corriger la tâche et d'institutionnaliser les résultats correspondants (cf. Extrait 2). Cette tendance apparaît dans une majorité d'épisodes $\mathrm{N}-1$ : ainsi le taux de questions est supérieur à $50 \%$ dans 13 épisodes sur 17 dans le jeu S4 J9 et inférieur à $50 \%$ dans six épisodes sur sept dans le jeu S4 J10.

Enfin, on peut remarquer que les élèves posent peu de questions, même si certains utilisent cette possibilité, particulièrement dans le jeu S4 J9 (cf. Extrait 3).

\section{Extrait 3}

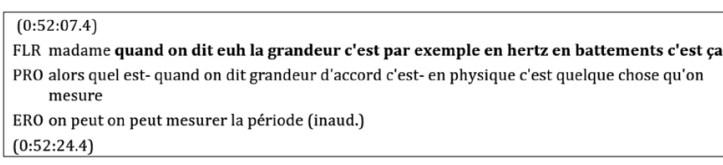

L'élève pose une question au professeur pour mieux comprendre l'énoncé de l'activité, mais ces questions sont rares (S4 J9 E.f).

\section{Dimension représentationnelle}

Pour caractériser la dimension représentationnelle des actes, nous les examinons d'abord sous l'angle du contenu des savoirs abordés, ensuite sous l'angle de la modélisation dans laquelle ils sont impliqués, et enfin sous l'angle des tâches épistémiques

Tableau 7 : Répartition pour le professeur et les élèves de la nature interactionnelle de leurs interventions dans les interactions (Analyse au niveau N-2)

\begin{tabular}{|c|c|c|c|c|c|}
\hline & \multicolumn{3}{|c|}{$\begin{array}{c}\text { Professeur } \\
\text { Jeu S4 J9: N = 133 }\end{array}$} & \multicolumn{2}{c|}{$\begin{array}{c}\text { Élèves } \\
\text { Jeu S4 J9: N = 100 } \\
\text { Jeu S4 J10 : N = 77 }\end{array}$} \\
\hline & $\begin{array}{c}\text { Juestion } 10: N=157 \\
\text { Injonction }\end{array}$ & Déclaration & $\begin{array}{c}\text { Poursuite } \\
\text { évaluative }\end{array}$ & Réponse & Question \\
\hline Jeu 9 & $77-58 \%$ & $19-14 \%$ & $27-20 \%$ & $86-86 \%$ & $6-6 \%$ \\
\hline Jeu 10 & $63-40 \%$ & $48-31 \%$ & $43-27 \%$ & $72-94 \%$ & $2-3 \%$ \\
\hline
\end{tabular}


qu'ils mettent en jeu. De fait, un contenu, une tâche de modélisation, une tâche épistémique, peut être développé sur un seul acte ou sur deux ou trois. Nous analysons donc ici des actes ou des groupes très réduits d'actes.

\section{Contenu abordé dans les actes ou groupes} d'actes

Lors de la structuration de l'action didactique en épisodes N-1 des jeux S4 J9 et S4 J10, basée sur les évolutions des trois dimensions de l'action didactique, nous avions montré qu'un réseau conceptuel associé aux caractéristiques des phénomènes périodiques constituait l'enjeu des interactions. La caractérisation microscopique du contenu du savoir confirme cette première analyse. Elle révèle en effet l'existence :

- de facettes liées directement à ce réseau conceptuel : celles-ci sont rattachées aux catégories thématiques "phénomène périodique », " période », " fréquence », « cycle motif », " mesure (sur le graphe) », « relation cycle - durée - période » et sont largement majoritaires

- de facettes liées à la situation proposée pour la tâche (exploitation d'un ECG) : celles-ci sont rattachées aux catégories " grandeur physique », «tension/volt », « représentation graphique » et «phénomène cardiaque » et sont donc minoritaires dans les interactions des deux jeux.

La répartition entre les deux types de facettes montre que les propos tenus dans la classe aussi bien lors des interventions du professeur auprès des petits groupes (jeu S4 J9) que de lors de la correction (jeu S4 J10) concernent beaucoup moins la compréhension des éléments de la situation comme le graphe et ses coordonnées (questions 1 et 2 voir annexe 1 ) que la détermination de la période et la fréquence des signaux. En effet, plus de la moitié des facettes sont relatives au réseau conceptuel dans le jeu S4 J9 et près des trois quarts dans le jeu S4 J10.

Tâches de modélisation mises en jeu dans les actes ou groupes d'actes

Le Tableau 8, qui rapporte la répartition entre professeur et élèves des actes ou groupes d'actes examinés sous l'angle de la modélisation, montre que les élèves prennent une part prépondérante dans ces tâches ( $81 \%$ vs $19 \%$ pour le professeur dans le jeu S4 J9 et $65 \%$ vs $35 \%$ dans le jeu S4 J10), et ce, qu'il s'agisse de décrire des éléments des théories ou modèles, des observables, ou de mettre les théories/ modèles et observables en relation. On peut d'ailleurs souligner l'importance de la contribution des élèves aux tâches de modélisation dans la correction de l'activité (jeu S4 J10), qui ne peut que s'appuyer sur leur travail dans les petits groupes, majoritairement en autonomie. Ainsi le travail des élèves sur le savoir dans le jeu S4 J9 contribue fortement au jeu S4 J10. La prépondérance des élèves dans les tâches de modélisation est avérée dans 11 épisodes sur 17 pour le jeu S4 J9 et dans les sept épisodes du jeu S4 J10. On peut aussi remarquer (cf. Tableau 8 ou Tableau 9) que dans le jeu S4 J9 qui se déroule en groupes de 2, ni professeur ni élève ne mettent en jeu la description d'objets et d'évènements. Rappelons que nous avons considéré que les graphes des électrocardiogrammes jouaient ici le rôle d'objets sur lesquels le professeur et les élèves travaillent. Dans ce jeu, les élèves décrivent les aspects conceptuels en lien avec les « demandes d'agir » / " questions - injonctions » du professeur signalées dans les analyses pragmatiques et interactionnelles (voir Extrait 1) et les mettent ensuite en relation avec le champ empirique; globalement, cela traduit l'importance des définitions des constituants du réseau conceptuel dans le déroulement du travail en groupe.

Tableau 8 : Répartition des actes ou groupes d'actes entre professeur et élèves au regard de la modélisation

(Analyse au niveau N-2)

\begin{tabular}{|c|c|c|c|c|c|c|c|c|c|}
\hline & \multicolumn{2}{|c|}{ Théories Modèles } & \multicolumn{2}{|c|}{ Observables } & \multicolumn{2}{|c|}{$\begin{array}{c}\text { Mise en relation Th. / } \\
\text { Mod. et Obs. }\end{array}$} & \multirow[t]{2}{*}{ Total } & \multirow[t]{2}{*}{$\%$ Prof. } & \multirow[t]{2}{*}{ \% Élèves } \\
\hline & Prof. & Élèves & Prof. & Élèves & Prof. & Élèves & & & \\
\hline Jeu S4 J9 & 4 & 14 & 0 & 0 & 6 & 28 & 52 & $10-19 \%$ & $42-81 \%$ \\
\hline Jeu S4 J10 & 5 & 16 & 14 & 23 & 10 & 27 & 95 & $29-35 \%$ & $66-65 \%$ \\
\hline
\end{tabular}

Rappelons qu’il est question de modélisation lorsque les élèves ou le professeur décrivent des théories - modèles, des observables ou les mettent en relation lorsqu'ils effectuent des tâches d'interprétation ou de prédiction. 
Tableau 9 : Répartition des actes ou groupes d'actes selon les tâches de modélisation qu'ils mettent en jeu

(Analyse au niveau N-2)

\begin{tabular}{|c|c|c|c|}
\hline & $\begin{array}{l}\text { Théories } \\
\text { Modèles }\end{array}$ & Observables & $\begin{array}{c}\text { Mise en } \\
\text { relation Th. } \\
/ \text { Mod. et } \\
\text { Obs. }\end{array}$ \\
\hline Jeu S4 J9 ; N = 52 & $18-35 \%$ & $0-0 \%$ & $34-65 \%$ \\
\hline Jeu S4 J10 ; N = 95 & $21-22 \%$ & $37-39 \%$ & $37-39 \%$ \\
\hline
\end{tabular}

Quant au jeu S4 J10, lors de la phase de correction de l'activité, les tâches de modélisation sont plus équilibrées (cf. Tableau 9) : le professeur et surtout les élèves qui y contribuent grandement sous l'égide du professeur (cf. Extrait 4), effectuent la description du champ empirique, la description du champ théorique pour ensuite les mettre en en relation.

Extrait 4

\begin{tabular}{|l|} 
[1:16:09.4) \\
PRO qu'est-ce que la fréquence \\
MIC c'est le nombre de battements sur un temps donné \\
PRO c'est le nombre de battements sur un temps donné donc tu peux le faire effectivement mais tu \\
$\quad$ vas- tu vas pas l'avoir très précisément et surtout tu l'auras pas en hertz or là vous venez de me \\
dire que votre graphique il représentait une tension en fonction de quoi \\
ELX du temps \\
PRO du temps donc est-ce qu'on peut pas se servir de cet axe des temps euh LEN \\
LEN on peut se servir de la enfin ce qu'on peut repérer tout de suite c'est la période \\
(1:17:39.6) \\
\hline
\end{tabular}

Lors de la correction, professeur et élèves mettent en ouvre différentes composantes de la modélisation (S4 J10 Ep 4).

Tâches épistémiques

Le Tableau 10 qui récapitule les tâches épistémiques mises en ouvre et leur répartition entre professeur et élèves permet de dégager plusieurs éléments.

Tout d'abord, ici aussi, on remarque la contribution importante des élèves au regard de celle du professeur dans les tâches liées à la manipulation du savoir, y compris dans la phase de correction de l'activité (48 vs 21 soit pratiquement $70 \%$ des tâches mises en jeu). L'activité épistémique des élèves est d'ailleurs d'autant plus significative que nous ne comptabilisons pas dans le jeu S4 J9 tout ce qui se fait en autonomie dans les petits groupes hors présence de l'enseignant. Cette tendance est observée dans 13 épisodes du jeu S4 J9 sur 17 et six épisodes du jeu S4 J10 sur sept.

Ensuite, deux types de tâches prédominent nettement, la description et l'interprétation. L'interprétation est majoritaire dans le jeu S4 J9 (cf. analyse précédente sur les tâches de modélisation). En petits groupes, le professeur invite les élèves à rappeler des définitions des éléments du réseau conceptuel, puis à les appliquer pour interpréter des éléments du champ empirique comme on peut le voir dans l'Extrait 5).

\section{Extrait 5}

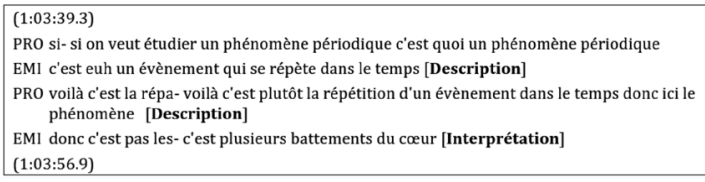

Le professeur demande à un groupe de préciser ce qu'est un phénomène périodique. L'élève répond, puis le professeur reformule sa proposition (dans les deux cas, descriptions théoriques) ce qui permet à l'élève de mettre en relation l'espace théorique, le concept de phénomène périodique et l'espace empirique, l'électrocardiogramme qu'il interprète (S4 J9 Ep n).

Dans le jeu S4 J10, les descriptions sont plus nombreuses ${ }^{9}$, certainement parce que concernant à la fois les champs empiriques (les électrocardiogrammes) et théoriques (les éléments du réseau conceptuel «phénomène périodique) (cf. Tableau 9). Les interprétations sont associées à des mises en relation entre les définitions des concepts et les électrocardiogrammes ou les battements de cœur.

Tableau 10 : Nombre des tâches épistémiques mises en jeu selon leur nature et l'acteur qui la réalise

(Analyse au niveau N-2)

\begin{tabular}{|c|c|c|c|c|c|c|c|c|c|c|c|}
\hline & \multicolumn{4}{|c|}{ Professeur } & \multicolumn{4}{|c|}{ Élève } & \multicolumn{3}{|c|}{$\begin{array}{c}\text { Total } \\
\text { S4 J9 : } \mathrm{N}=50 ; \mathrm{S} 4 \mathrm{~J} 10: \mathrm{N}=69\end{array}$} \\
\hline & 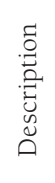 & 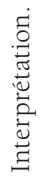 & 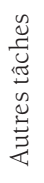 & 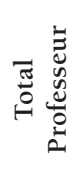 & 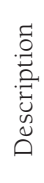 & 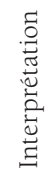 & 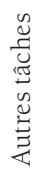 & 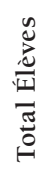 & 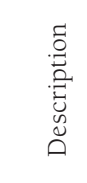 & 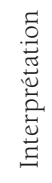 & 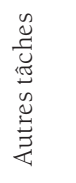 \\
\hline jeu S4 J9 & 3 & 5 & 1 & 9 & 14 & 20 & 7 & 41 & 17 & 24 & 8 \\
\hline jeu S4 J10 & 12 & 5 & 4 & 21 & 28 & 15 & 5 & 48 & 40 & 20 & 9 \\
\hline
\end{tabular}


Enfin, dans les « autres tâches épistémiques » qui sont marginales, on trouve « mesurer» « comparer» et « argumenter». Il y a donc que peu ou pas d'argumentation dans le jeu S4 J10 et la partie " publique » du jeu S4 J9. Ceci renforce l'idée que le professeur décide du savoir mis en jeu et oriente généralement sa mise en œuvre par les élèves, en tenant des propos dans lesquels ils s'insèrent. L'importante contribution des élèves à l'avancée du savoir n'est donc pas forcément liée à un questionnement autonome sur le savoir.

\section{Description de modalités de l'action didactique au sein de chaque jeu (niveau mésoscopique $\mathrm{N}$ )}

L'ensemble des analyses précédentes (structuration globale et analyse microscopique) montre une homogénéité importante des épisodes $\mathrm{N}-1$ (échelle 1-5 minutes) appartenant à chacun des jeux. Celle-ci nous a déjà autorisés à récapituler les analyses microscopiques directement au niveau mésoscopique $\mathrm{N}$ de chaque jeu et non pas au niveau N-1 de chacun de ces épisodes. Elle nous permet aussi de tracer les contours de l'action didactique à l'échelle de chaque jeu, en nous centrant sur les conditions dans lesquelles le savoir avance dans chacun d'eux et en prenant en compte simultanément l'ensemble des dimensions pragmatique, interactionnelle et représentationnelle des actes. Si cette séparation des dimensions facilite l'analyse, c'est bien en recombinant aux échelles supérieures les résultats relatifs à chacune d'entre elles qu'on trouve du sens, un acte étant porteur en même temps souvent des trois dimensions.

Dans le jeu S4 J9, des sollicitations du professeur auxquelles les élèves souscrivent selon leurs connaissances et leur compréhension de la tâche

Nos analyses précédentes montrent les ressorts de l'action didactique conjointe dans le jeu S4 J9. Le professeur sollicite les élèves pour qu'ils élaborent ou approfondissent leurs propositions, les élèves décrivent et interprètent, ils contribuent majoritairement au développement du savoir. Ainsi dans l'Extrait 1 on voit le professeur demander à un groupe comment il a compris la question sur laquelle il travaille. La validation de la réponse en partie complétée par le professeur conduit les élèves à faire une proposition, mais celle-ci est erronée. Le professeur poursuit alors en demandant de définir la période, ce qui aide l'élève à formuler une réponse pertinente. De manière plus générale, on peut dire que les connaissances mobilisées par les élèves à partir de la sollicitation du professeur correspondent à un savoir relatif au réseau conceptuel « phénomène périodique » récemment enseigné et donc souvent approximatif. Ils sont autorisés à le formuler en l'état et les sollicitations successives du professeur leur permettent de le préciser et de l'utiliser eux-mêmes pour avancer dans la tâche. Cette lecture de l'action didactique trouve son origine dans la cohérence qui existe entre l'importance des « demandes d'agir» du professeur à travers ses questions ou injonctions, les concepts qu'il choisit de proposer à la discussion dans ses interventions, l'importance de « l'agir » des élèves dans le cadre de leurs réponses, leur contribution prépondérante dans les tâches de modélisation et les tâches épistémiques, éléments tous révélés dans l'analyse microscopique.

Pour compléter ce tableau fourni par l'analyse microscopique, on peut rajouter deux caractéristiques complémentaires : d'une part, c'est généralement le professeur qui oriente le tout (au moins dans les moments où il est présent), l'élève s'il contribue fortement, le fait la plupart du temps sous le contrôle de l'enseignant et ne fait quasiment pas preuve d'initiative dans les échanges; d'autre part, les élèves (ou du moins une majorité) ont compris les enjeux de l'activité et les données proposées. En effet, le professeur n'intervient pas pour décrire les graphes de l'électrocardiogramme qui sont considérés ici comme relevant du domaine empirique. Il intervient principalement pour aider à leur interprétation en termes de motif, période ou fréquence et de temps en temps pour reformuler les définitions conceptuelles.

\section{Dans le jeu S4 J10, une très forte densité épistémique liée aux interventions du professeur nourries par celles des élèves et appuyées sur le travail dans le jeu précédent}

En revanche dans le jeu S4 J10, la répartition s'inverse sur plusieurs plans. Les demandes d'agir, toujours présentes sont moindres, le professeur agit lui-même davantage, notamment en termes de déclaration et d'évaluation, il reprend explici- 
tement la description des graphes, précise les définitions (grandeur, période, fréquence) et explicite donc tous les éléments utiles pour réaliser la tâche. Par ses déclarations et ses poursuites évaluatives, il explicite les résultats attendus et garantit leur pertinence à des fins d'institutionnalisation. Ces éléments se traduisent par une " densité épistémique » du jeu plus importante (16 actes liés au savoir à la minute vs 10 dans le jeu S4 J9), associée principalement à l'augmentation du nombre d'actes du professeur (11 vs 6 actes/min) alors que la densité des interventions des élèves est à peu près constante (5 vs 4 actes / min). Ainsi dans ce jeu, le professeur développe un grand nombre d'actes liés au savoir en peu de temps: nous avons relevé jusqu'à 65 actes liés au savoir pour 18 tours de parole, un tour de parole pouvant en compter jusqu'à 5 comme le montre l'Extrait 6 . On y voit le professeur, à la suite d'une proposition d'un élève de mesurer la fréquence directement sur l'électrocardiogramme, le solliciter pour définir ce qu'on entend par fréquence puis à la suite de sa réponse, enchaîner dans son tour de parole, cinq actes consécutifs tous en lien avec le savoir. Après la réponse de l'élève, il poursuit par une évaluation, déclare que la mesure est effectivement possible, qu'elle ne sera pas précise, qu'elle ne sera pas en Hertz, et enfin demande à l'élève de faire une proposition quant à l'unité (seconde) présente sur le graphe, susceptible de l'amener à penser que c'est la période et non la fréquence qu'on peut lire directement.

Extrait 6

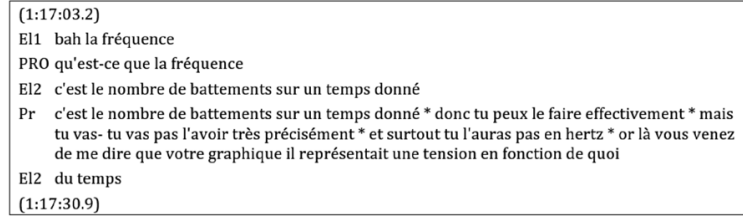

Dans le jeu S4 J10, la densité épistémique est plus importante que dans le jeu $\mathrm{S4} \mathrm{J} 9$, en raison de la richesse épistémique des interventions du professeur (chaque partie correspondant à un acte est séparée par un astérisque) (S4 J10 Ep 4).

Cet extrait montre aussi que les interventions des élèves enrichissent celles du professeur du point de vue du savoir et de son fonctionnement. Ainsi, les contributions des élèves à ce jeu ne sont possibles que grâce à un travail centré sur l'enjeu de l'activité. Comme dans la section précédente, les conclusions tirées quant au jeu S4 J10 sont en cohérence avec les résultats de l'analyse microscopique, par exemple la diminution des « demandes d'agir » et questions du professeur au profit « d'un agir personnel » en termes de déclarations et d'évaluations, l'importance des réponses dans l'agir de l'élève, l'importance des tâches de modélisation et des tâches épistémiques qu'il assume dans ce jeu.

\section{Composantes du contrat établies au niveau $\mathrm{N}$}

L'ensemble des analyses précédentes interprétées au niveau mésoscopique, et en particulier la description des pratiques développée dans la section "Description de modalités de l'action didactique au sein de chaque jeu (niveau mésoscopique N)", nous conduisent à faire les inférences suivantes sur le contrat relatif à cette activité (cf. phase 2 - étape 3 de la Figure 8). La première concerne le jeu introduisant l'activité, les autres concernent les deux jeux principaux (jeux S4J9 et S4 J10), certaines sont communes aux deux jeux et les autres spécifiques de l'un ou de l'autre. Ces composantes sont exprimées sous la forme de règles à suivre par les différents acteurs, étant entendu que chacun s'attend à ce que l'autre les respecte.

\section{Composante relative au jeu S4 J8 introductif à}

l'activité

Au vu de la durée du jeu introductif au cours duquel le professeur donne le titre, distribue les documents en classe entière et demande aux élèves de se mettre au travail, ce qu'ils font effectivement, on peut dire que :

- Les élèves doivent se mettre rapidement au travail en groupe de deux après une introduction très brève de l'activité et la distribution des documents associés.

\section{Composantes communes aux jeux S4 J9 et S4 J10}

Trois règles communes aux deux jeux principaux ont une grande importance dans le déroulement de l'action didactique :

- Professeurs et élèves ont essentiellement à traiter le savoir, enjeu de l'activité.

- Les élèves ont à contribuer au savoir de la classe.

- Le professeur prend souvent l'initiative du savoir à traiter dans les échanges et généralement les oriente. 
Trois autres règles viennent préciser les précédentes:

- Les élèves doivent s'approprier le réseau conceptuel relatif à « phénomène périodique ». Cette règle spécifie le savoir enjeu de l'activité.

- Les élèves doivent répondre aux nombreuses questions ou injonctions du professeur. Cette règle spécifie à la fois la manière dont s'effectue le pilotage du professeur et la manière dont les élèves contribuent au savoir - Les élèves ont la possibilité de poser des questions. Cette règle spécifie la manière dont les élèves agissent quand ils orientent (rarement) le déroulement de l'action didactique.

Composantes spécifiques au jeu S4 J9 (réalisation de la tâche en groupes de deux)

- Les élèves doivent travailler en groupe de façon autonome sur l'enjeu du savoir visé dans l'activité, en mobilisant leurs acquis

- Les élèves doivent écrire leurs réponses qui peuvent être correctes ou incorrectes

- Le professeur doit solliciter les élèves à partir des difficultés et des erreurs dans le processus de résolution qu'il identifie dans les productions écrites de chaque groupe, ou plus rarement dans les questions qu'ils posent.

- Lors des interventions du professeur, les élèves doivent être en mesure de rapporter l'état de leur travail et doivent mobiliser leurs connaissances sur les définitions des concepts introduites récemment.

- Le professeur doit accepter les propositions plus ou moins correctes ou précises des élèves, et les inciter par ses questions ou commentaires à aller au-delà dans leur explicitation et dans leur mobilisation dans l'interprétation des observables du champ empirique.

- Les élèves doivent ensuite poursuivre seuls leur travail à partir des supports de l'activité (ici les ECG et l'énoncé de la tâche à réaliser). Ce travail en autonomie doit leur permettre de participer à la phase de correction de l'activité 4 .

Composantes spécifiques au jeu S4 J10 (phase de correction de l'activité en classe entière)

- Le professeur doit demander aux élèves de contribuer au savoir, ce qu'ils font, et doit y contribuer lui-même de manière dense (en conséquence ceci suppose que les élèves soient particulièrement attentifs s'ils veulent suivre ce que dit le professeur et répondre à ses sollicitations).

- Le professeur doit reprendre chacune des questions de l'activité, expliciter et surtout faire expliciter par les élèves l'ensemble des données empiriques ainsi que les éléments théoriques du savoir et leur mise relation. Il garantit la pertinence des réponses et les institutionnalise.

\section{Contrat lié au déroulement de l'activité 4 établi à l'échelle N+1 (1h à $1 \mathrm{~h} 30)$}

Après avoir inféré les règles du contrat à l'échelle mésoscopique (niveau $\mathrm{N}$ ), nous complétons maintenant les règles relatives à l'activité 4 en examinant l'action didactique à l'échelle $\mathrm{N}+1$ de $1 \mathrm{~h}$ à $1 \mathrm{~h} 30$. C'est l'étape 4 présentée dans la Figure 8 synthétisant la méthode d'investigation.

Rappelons que l'activité 4 est structurée en trois jeux (cf. Tableau 11) et qu'elle porte sur l'exploitation de deux électrocardiogrammes pour identifier et déterminer les grandeurs caractéristiques (fréquence et période) des battements cardiaques. L'identification et la détermination de ces caractéristiques ont été menées, d'abord en prenant connaissance rapidement et collectivement de la tâche à réaliser, puis en construisant en groupe de deux à partir de ce que les élèves savent déjà, des idées permettant de répondre aux questions posées, et enfin, à nouveau collectivement, lors de la correction en formalisant et institutionnalisant les solutions correspondantes en prenant appui sur ces idées.

Comme nous l'avons déjà montré dans l'analyse en actes, le travail en groupe (S4J9) nourrit le jeu suivant de mise en commun et d'institutionnalisation. Ce travail en groupe apparaît donc comme doublement important dans cette séquence, pour l'élève qui va s'appuyer dessus pour comprendre la formalisation établie en classe entière par le professeur, et pour le professeur qui utilise les contributions des élèves pour produire ses institutionnalisations. Il est donc important que ce travail en groupe soit effectif (engagement des élèves dans la tâche) et suffisamment productif (aide suffisante du professeur). 
Tableau 11 : Structuration de l'action didactique relative à l'activité 4 à l'échelle mésoscopique (Niveau N) en trois jeux

\begin{tabular}{|l|l|l|l|}
\hline S4 J8 & $\begin{array}{l}\text { Prendre connaissance de l'acti- } \\
\text { vité proposée visant à exploiter } \\
\text { deux électrocardiogrammes } \\
\text { pour identifier et déterminer } \\
\text { leurs grandeurs caractéristiques }\end{array}$ & $\begin{array}{l}\text { Classe } \\
\text { entière }\end{array}$ \\
\hline S4 J9 & $\begin{array}{l}\text { En collaborant avec son parte- } \\
\text { naire, exploiter deux électrocar- } \\
\text { diogrammes (ECG) pour identi- } \\
\text { fier et déterminer ses grandeurs } \\
\text { caractéristiques (fréquence } \\
\text { cardiaque période) }\end{array}$ & $\begin{array}{l}\text { Petit } \\
\text { groupe }\end{array}$ \\
\hline S4 J10 & $\begin{array}{l}\text { Comprendre grâce à la discus- } \\
\text { sion collective la correction de la } \\
\text { détermination de la fréquence et } \\
\text { la période de deux ECG. }\end{array}$ & $\begin{array}{l}\text { Classe } \\
\text { entière }\end{array}$ \\
\hline
\end{tabular}

Dans ces jeux, successivement, les élèves avaient à prendre connaissance de la tâche, à la résoudre en produisant de nouvelles idées en groupe de deux, puis à comprendre la correction collective correspondante à des fins d'institutionnalisation.

Aussi trois nouvelles composantes du contrat apparaissent à cette échelle :

- La résolution de la tâche proposée dans l'activité s'opère en trois phases : prise de connaissance de la tâche, réalisation collaborative partielle ou totale de la tâche par groupe de deux, correction en classe entière basée sur le travail de groupe et institutionnalisation par le professeur.

- Lélève doit être systématiquement engagé avec son/sa partenaire dans la réalisation de la tâche donnée.

- Le professeur doit aider chaque groupe à progresser suffisamment dans la réalisation de chaque tâche.

La suite des résultats concerne la $3^{\mathrm{e}}$ phase de l'investigation, dans laquelle il s'agit de tester la validité de ces règles formulées à l'échelle $\mathrm{N}$ et $\mathrm{N}+1$ dans les autres activités de la séquence, et éventuellement de les compléter.
Figure 10 : les sections suivantes rapportent les résultats issus de la phase 3 de la méthode d'investigation

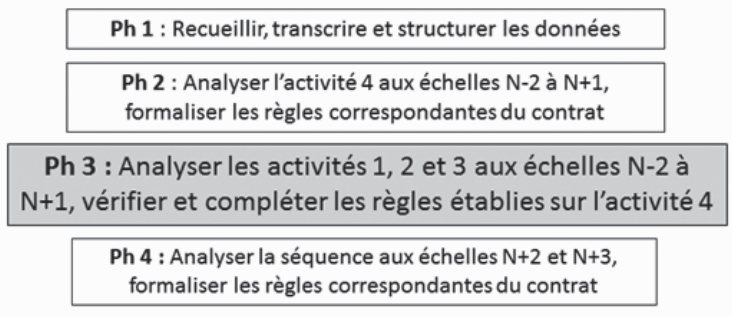

STABILITÉ DU CONTRAT ÉTABLI SUR L'ACTIVITÉ 4 SUR L'ENSEMBLE DE LA SÉQUENCE

Le contrat dont nous venons de préciser certaines composantes aux niveaux $\mathrm{N}$ et $\mathrm{N}+1$ semble bien établi à la fin de la séquence, lors du déroulement de l'activité 4. Mais cette séquence d'enseignement est nouvelle pour la professeure et ses élèves. Aussi, la question de sa stabilité tout au long de la séance se pose-t-elle : ce contrat, est-il établi très rapidement dès le début de la séquence, ou évolue-t-il au cours de la séquence pour aboutir à ce qu'il est dans l'activité 4? Nous envisageons tour à tour la stabilité des composantes du contrat au niveau $\mathrm{N}$, puis au niveau $\mathrm{N}+1$.

\section{Stabilité des composantes établies à l'échelle mésoscopique $\mathrm{N}$}

Comme nous l'avons expliqué dans la partie méthodologique, nous n'avons pas fait d'analyse systématique des trois autres activités au niveau microscopique. Nous avons d'abord évalué si les caractéristiques des actes en lien avec les trois règles communes aux jeux principaux de l'activité 4 , jugées comme importantes, étaient majoritaires dans chaque épisode N-1 des activités 1,2 et 3 . Nous avons ensuite réalisé une analyse thématique pour confirmer ou non l'existence de toutes les autres règles. Nous présentons tour à tour dans cette partie les analyses relatives :

- à la règle spécifique aux jeux introductifs

- aux règles communes à l'ensemble des jeux et principalement celles qui sont relatives aux trois règles les plus importantes;

- aux règles spécifiques à la réalisation de la tâche en groupe de deux ; 
- aux règles spécifiques à la correction de la tâche et l'institutionnalisation des conclusions en classe entière.

\section{Règle relative aux jeux introductifs}

Comme dans l'activité 4, les trois activités 1, 2 et 3 sont introduites très succinctement en classe entière : les jeux correspondants sont très courts ( 2 à 5 minutes) et constitués de seulement 1 ou 2 épisodes N-1 (voir par exemple dans le Tableau 12 la durée du jeu 5). Le professeur demande de lire le texte distribué, quelquefois même partiellement en se limitant à l'objectif de l'activité, il peut parfois répondre à une ou deux questions si bien que dans les quatre activités, les élèves doivent se mettre rapidement au travail en groupe de deux après une introduction très brève ${ }^{10}$ (cf. par exemple l'Extrait 7 issu de l'activité 2). Ils ont donc à leur charge de lire et comprendre ce qui leur est demandé.

Extrait 7

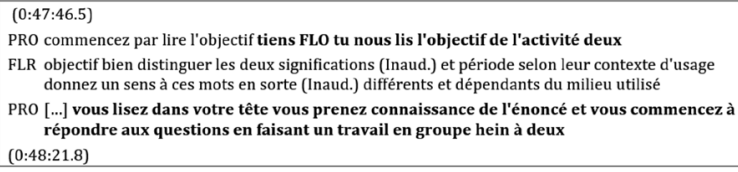
donnez un sens à ces mots en sorte (Inaud.) différents et dépendants du milieu utilisé

PRO [...] vous lisez dans votre tête vous prenez connaissance de l'énoncé et vous commencez à répondre aux questions en faisant un travail en groupe hein à deux

$(0: 48: 21.8)$

Le professeur fait prendre connaissance aux élèves de l'activité à réaliser a minima, et leur demande de la réaliser en groupes (S2 J11 / activité 2)

\section{Règles communes à l'ensemble des jeux principaux}

Rappelons d'abord les trois règles importantes dans la classe, communes aux jeux principaux de l'activité 4 : les actes du professeur et des élèves sont essentiellement centrés sur le savoir, les élèves ont à contribuer au savoir et le professeur prend souvent l'initiative du savoir et généralement oriente les échanges, et l'élève y prend une part significative. Nous avons évalué leur validité en examinant dans chaque épisode des activités 1, 2 et 3 si la majorité des actes qui les constituent ont des caractéristiques en accord avec elles. Nous avons récapitulé ces résultats pour chaque jeu au sein de tableaux identiques au Tableau 12 qui lui, concerne spécifiquement l'activité 3.
Dans cette activité expérimentale (cf. la suite de jeux du Tableau 12), les élèves ont pour tâche de déterminer la période et la fréquence d'un hautparleur connecté à un générateur basse fréquence en imaginant comment procéder ${ }^{11}$. Ils doivent ensuite formaliser le protocole correspondant à l'extérieur de la classe, le professeur ayant expliqué dans la séance 3 la manière de rédiger un protocole en physique. La correction du protocole est opérée en classe entière à partir d'une photocopie distribuée par le professeur. Les élèves ont à la compléter par les calculs de fréquence et de période à partir du nombre de vibrations qu'ils ont comptées pendant une durée déterminée lors de l'investigation.

En excluant le jeu introductif S2 J5 de nos commentaires dédiés aux jeux principaux, il apparaît que dans un jeu (S2 J6), les épisodes N-1 dans lesquels le savoir occupe la part principale des interactions sont peu nombreux : la mise au travail en autonomie, même si elle est demandée par le professeur dans le jeu précédent, n'est pas immédiatement effective. Le professeur est contraint de présenter la manière d'utiliser le matériel mis à disposition des élèves, c'est-à-dire contraint de construire la composante matérielle du milieu, permettant de travailler ensuite de façon autonome. Un phénomène de même type survient dans l'activité 1 au cours de laquelle les élèves ont à prendre connaissance de trois mouvements pour ensuite décider, d'abord en groupe de deux puis collectivement, lesquels sont périodiques. Deux de ces mouvements sont présentés en vidéo et ne sont donc pas disponibles en permanence pour la réflexion. Les élèves au cours de leur travail en groupes, demandent donc régulièrement au professeur de revisionner ces mouvements mais aussi des précisions sur les mots de l'énoncé de la tâche. En d'autres termes, le milieu adéquat n'est pas complètement disponible quand les élèves commencent à réaliser la tâche, si bien que le professeur est amené à le préciser presque tout au long du travail de groupe supposé être en autonomie, et l'organisation de la classe est alors mixte (groupe et classe entière). Le nombre d'épisodes centrés sur le savoir est là aussi minoritaire. Ces deux cas nous conduisent à ajouter une nouvelle règle à celles mises en évidence dans l'activité 4 , cruciale lorsque les élèves travaillent en autonomie : le professeur doit rendre effectif pour les élèves les éléments nécessaires à la tâche ou, en termes plus généraux, "le professeur doit rendre disponible aux élèves un milieu adéquat à la réali- 
Tableau 12 : Résultats de l'analyse des épisodes de l'activité 3

\begin{tabular}{|c|c|c|c|c|}
\hline Jeux relatifs au déroulement de l'activité 3 & $\begin{array}{l}\text { Organisat. de } \\
\text { classe }\end{array}$ & $\begin{array}{l}\text { Épisodes N-1 } \\
\text { où Savoir } \\
\text { central }\end{array}$ & $\begin{array}{l}\text { É p i s o des } \\
\mathrm{N}-1 \text { où Élève } \\
\text { contribue }\end{array}$ & $\begin{array}{l}\text { Épisodes } \mathrm{N}-1 \\
\text { où } \mathrm{P} \text { oriente }\end{array}$ \\
\hline $\begin{array}{l}\text { Jeu S2 J5: Se représenter collectivement la tâche «Trouver un } \\
\text { moyen pour déterminer expérimentalement la période et la } \\
\text { fréquence de vibration de la membrane d'un haut-parleur et le } \\
\text { mettre en œuvre» }(05: 07)\end{array}$ & $\begin{array}{l}\text { Classe } \\
\text { entière }\end{array}$ & $1 / 2$ & $1 / 1$ & $2 / 1$ \\
\hline $\begin{array}{l}\text { Jeu S2 J6 : Mettre en marche le dispositif (Générateur + Haut- } \\
\text { Parleur - HP) }(05: 19)\end{array}$ & Groupe de 2 & $2 / 6$ & $0 / 2$ & $4 / 2$ \\
\hline $\begin{array}{l}\text { Jeu S2 J7 : En collaborant avec son partenaire, trouver un moyen } \\
\text { pour déterminer expérimentalement la période et la fréquence de } \\
\text { vibration de la membrane d'un haut-parleur et le mettre en œuvre. } \\
(36: 40)\end{array}$ & Groupe de 2 & $17 / 21$ & $17 / 17$ & $12 / 17$ \\
\hline $\begin{array}{l}\text { À l'extérieur de la classe : préparation du protocole de l'investiga- } \\
\text { tion en collaborant à } 2 \text {, rédaction individuelle après que P dans la } \\
\text { séance } 3 \text { ait expliqué comment procéder pour rédiger un protocole } \\
\text { en cours de physique. }\end{array}$ & & & & \\
\hline $\begin{array}{l}\text { Jeu S4 J4 : Grâce à la discussion collective et avec l'appui de la } \\
\text { correction photocopiée, comprendre les attendus liés au proto- } \\
\text { cole relatif à la détermination expérimentale de la période et la } \\
\text { fréquence de vibration de la membrane d'un HP }(06: 55)\end{array}$ & $\begin{array}{l}\text { Classe } \\
\text { entière }\end{array}$ & $1 / 2$ & $0 / 1$ & $1 / 1$ \\
\hline $\begin{array}{l}\text { Jeu S4 J5: Compléter individuellement le protocole distribué par } \\
\text { le professeur en exploitant les mesures faites en TP (nombre de } \\
\text { vibrations du HP pendant une durée déterminée) pour calculer la } \\
\text { fréquence en hertz et la période du phénomène }(01: 52)\end{array}$ & $\begin{array}{l}\text { Classe } \\
\text { entière / } \\
\text { Individuel }\end{array}$ & $3 / 3$ & $1 / 3$ & $3 / 3$ \\
\hline $\begin{array}{l}\text { Jeu S4 J6 : Comprendre grâce à la discussion collective la manière } \\
\text { dont on calcule la fréquence et la période à partir de la mesure du } \\
\text { nombre de vibrations de la membrane du HP sur une durée déter- } \\
\text { minée et compléter le protocole distribué. }(08: 22)\end{array}$ & $\begin{array}{l}\text { Classe } \\
\text { entière / } \\
\text { individuel }\end{array}$ & $2 / 2$ & $2 / 2$ & $2 / 2$ \\
\hline
\end{tabular}

Les cellules indiquent le nombre d'épisodes dans lesquels les actes du professeur et des élèves sont majoritairement conformes aux trois règles les plus importantes du contrat. Les cellules grises identifient les jeux où ces actes ne sont pas majoritairement conformes à certaines de ces règles. Le premier jeu S2 J5 est un jeu introductif et ne fait pas partie des jeux principaux dont il est question dans cette partie (Analyse aux niveaux N-1 et N).

sation en autonomie de la tâche ». Enfin, on note encore dans l'activité 2 lorsque les élèves travaillent en groupe de deux, des épisodes centrés sur la gestion de la classe qui disparaissent dans les activités suivantes. Nous y voyons la trace de la stabilisation progressive de la règle sur la prédominance des actes liés au savoir en contexte de travail de groupe. Cette stabilisation progressive n'existe pas dans les phases de correction en classe entière, la centration sur le savoir des actes du professeur et des élèves y étant effective dès le début de la séquence.

Si on revient sur l'activité 3 en considérant maintenant la contribution significative des élèves à l'avancée des savoirs, le Tableau 12 indique que l'élève y contribue peu dans trois jeux. Dans le jeu S2 J6 déjà évoqué, c'est le professeur qui reprend les réglages du GBF que certains élèves ont modifié ou explique où il faut observer (membrane du haut-parleur) dans les différents groupes pour compléter le milieu initial. Dans le jeu S4 J4, le professeur corrige non seulement le contenu de l'activité mais la forme que doit prendre le protocole car c'est la première fois que les élèves en réalisent un en physique et ils ne peuvent rien apporter sur ce plan. Il a choisi de distribuer une correction incomplète, que les élèves doivent compléter individuellement dans le jeu S4 J5. Dans le cadre d'une organisation mixte classe entière - travail individuel, le professeur apporte une aide à quelques élèves dont profite l'ensemble de la classe pour passer d'un nombre de vibrations pendant une durée déterminée (souvent 1 minute) à une fréquence en Hertz (nombre de vibrations par seconde), ce qui explique la faible contribution des élèves durant ce jeu très court. Dans les autres activités, la contribution de l'élève est toujours importante, notamment dans les phases de correction en classe entière, même si 
la contribution du professeur en termes d'actes est toujours majoritaire (par exemple dans l'activité 1 , les élèves contribuent significativement au savoir dans 8 épisodes sur 13 en groupes de deux et dans 4 épisodes sur 6 en classe entière).

Enfin, le Tableau 12 relatif à l'activité 3 montre que le professeur garde le plus souvent l'initiative du savoir à traiter et pilote les échanges. C'est aussi le cas dans les activités 1 et 2 sauf dans les épisodes du travail de groupe au cours desquels les élèves demandent l'information qui leur semble nécessaire pour traiter les questions qui leur sont soumises; ceci manifeste la réciproque "élève » de la règle de mise à disposition du milieu matériel par le professeur : les élèves le lui réclament.

Quant aux autres règles communes à l'ensemble des jeux principaux, tous les extraits qui suivent dans cette section, liés à l'analyse thématique que nous avons réalisée en lien avec les règles du contrat de l'activité 4 montrent d'une part, que "les élèves doivent répondre aux nombreuses questions ou injonctions du professeur » et d'autre part que « les élèves doivent s'approprier le réseau conceptuel relatif au "phénomène périodique" ». En effet, le concept de mouvement périodique apparaît dans l'Extrait 13 et l'Extrait 14, le concept de fréquence dans l'Extrait 11 et les concepts de période et de cycle dans l'Extrait 12 .

L'ensemble de cette analyse nous conduit à considérer qu'il y a une stabilisation progressive de l'activité 1 à l'activité 4 de deux composantes du contrat qui ne sont pas sans liens : la prépondérance des actes liés au savoir lors du travail en groupes, alors que celle-ci est manifeste lors de la phase de correction en classe entière dès l'activité 1 , et d'autre part, la mise en place du milieu adéquat avant le travail en autonomie pour que celui-ci puisse être effectivement initié. Il peut y avoir une dérogation à cette dernière règle de manière conjoncturelle du fait d'un matériel qui ne fonctionne pas, mais elle est nécessaire pour que les élèves soient en mesure de travailler en autonomie (avec une aide possible du professeur) sur l'enjeu du savoir visé dans l'activité.

Enfin, l'ensemble des jeux (à l'exclusion des jeux d'introduction de l'activité, très brefs) présente une homogénéité sur l'ensemble de la séquence au regard des trois règles importantes du contrat. Établies à partir d'analyses au niveau N-2 (repérage des actes associés) et $\mathrm{N}-1$ (repérage des épisodes dans lesquels ces actes sont majoritaires), vérifiées au niveau mésoscopique (comptage des épisodes N-1 dans lesquels elles sont vérifiées), ces règles seront donc aussi valides aux échelles supérieures ${ }^{12}$.

Nous décrivons maintenant les résultats de l'analyse thématique relative aux règles spécifiques à la résolution des tâches en groupes puis aux règles spécifiques aux phases de correction et d'institutionnalisation en classe entière

\section{Règles spécifiques à la résolution des tâches en groupes}

Lorsqu'ils ont pris connaissance de la tâche, dans toutes les activités, les élèves doivent travailler en groupe de façon autonome. En effet, dans tous les moments de travail en autonomie, le professeur demande aux élèves de collaborer avec leur partenaire, mais cette demande apparaît plus fortement dans l'activité 1 et au début de l'activité 2 au cours desquelles elle est explicitée. On y voit le professeur demander explicitement de collaborer avec son partenaire à six reprises en 36 minutes, par exemple au jeu S1 J6, il demande "vous êtes d'accord tous les deux ou pas? [28: 18]», ou encore au jeu S1 J12, «mais vous pouvez travailler à deux c'est même conseillé [54: 14]». Elle devient implicite par la suite.

Dans l'activité 4, lorsqu'ils travaillent en groupe, les élèves doivent réaliser la tâche « en mobilisant leurs acquis » et aussi en écrivant leurs réponses qui peuvent être correctes ou incorrectes. Cela suppose donc que les élèves construisent des idées relatives à l'enjeu de savoir, qu'elles soient justes ou fausses. Ce statut du savoir particulier au travail en groupe est précisé par le professeur au cours des deux premières activités, comme par exemple dans l'Extrait 8. Les indications sur le statut du savoir et en conséquence celui de l'erreur, n'apparaissent plus dans les activités 3 et 4 : les élèves ont accepté cette règle et la respectent. Quant à la demande d'écrire les réponses justes ou fausses en incluant le processus de résolution, elle est constante dans toutes les activités comme en témoigne l'Extrait 8. Le professeur explicite même à deux élèves, à propos de la rédaction relative à l'activité 3 conduite à l'extérieur de la classe, la conduite à tenir : " oui mais faire à deux c'est différent de recopier [...] faire à deux c'est on réfléchit ensemble et ensuite on fait par écrit ». 
Extrait 8

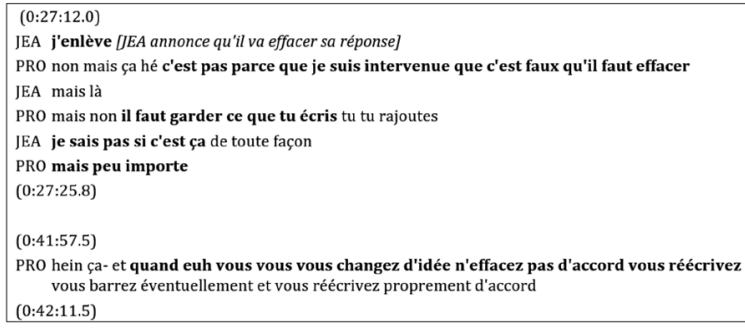

Lors du travail en petit groupe, le professeur demande à l'élève de ne pas effacer son raisonnement et son résultat, même si celui-ci est peut-être erroné (S1J7 Ep 8 - Activité 1 et S2 J5 - Activité 2).

Quant aux règles restantes concernant les sollicitations du professeur, les réponses des élèves et la suite qui leur est donnée, aucun acte du professeur ou des élèves ne vient les contredire tout au long de la séquence. Ainsi dans l'Extrait 9 emprunté à l'activité 3 , on peut ainsi voir que le professeur prend connaissance du processus de résolution dans la production écrite du groupe et qu'à partir de sa lecture, il sollicite les élèves qui mobilisent leurs acquis pour répondre, ici à propos de la définition d'un concept introduite récemment (la fréquence). Le professeur accepte les propositions approximatives ou incorrectes des élèves (l'élève propose une définition erronée sans qu'il fasse l'objet d'une remontrance), et les incite par ses questions ou commentaires à aller au-delà dans leur explicitation et dans leur mobilisation dans l'interprétation des observables du champ empirique (ici transformer la première proposition en battements par minute en Hertz). Comme dans l'activité 4 , les élèves ont ensuite à poursuivre seuls leur travail à partir des informations disponibles comme on le voit dans la dernière intervention du professeur dans l'Extrait 9.
Extrait 9

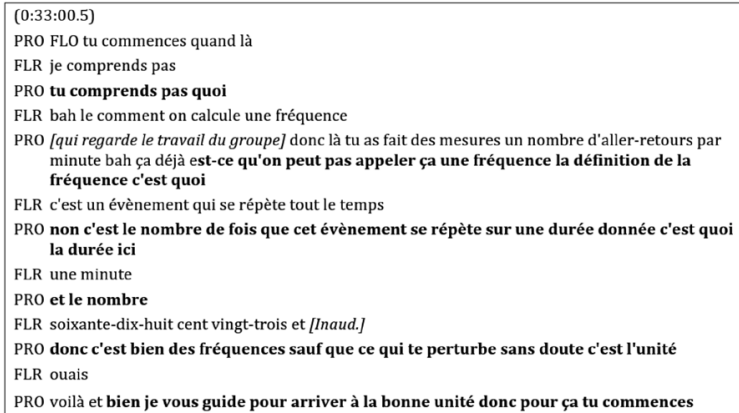

Lors du travail en petit groupe le professeur, après avoir pris connaissance de ce que le groupe a écrit, aide un groupe d'élèves à avancer dans la résolution de la tâche en demandant puis en précisant ce qu'est la fréquence. Les élèves ont à s'appuyer sur cette définition pour poursuivre leur travail et produire une réponse (S4 J5 Ep 3 - Activité 3).

Enfin, l'analyse des activités 2 et 3 met particulièrement en évidence l'importance que le professeur donne à la façon dont les élèves réalisent la tâche en groupes. Il donne la priorité à la démarche par rapport au résultat, ce qui apparaît par exemple dans l'Extrait 10, mais aussi dans le jeu S4 J4 (cf. Tableau 12) au cours duquel le professeur à travers la correction photocopiée du protocole indique aux élèves comment rendre compte d'une investigation mettant en jeu un aspect expérimental. Cela nous conduit à inférer une nouvelle règle du contrat qui traduit le fait que le fonctionnement du savoir est essentiel : résoudre une tâche en classe de physique c'est avant tout être capable de construire une démarche en utilisant des savoirs déjà enseignés et de l'expliquer, le résultat restant important mais pas suffisant. Cette priorité donnée à la démarche est présente dans l'activité 4 mais implicite, l'enseignant prenant connaissance du travail des élèves sans faire de remarque sur le sujet, ce qui montre que les élèves respectent cette règle à ce moment-là de la séquence.

Extrait 10

(1:10:32) (le professeur arrive auprès du group

JUL là du coup ça fait cinq sur vingt-quatre

PRO alors alors directement tu me poses ton résultat (rire) je veux savoir ce que vous avez fait $1: 10: 38.6$ )

Lors du travail en petit groupe le professeur signifie à un élève qu’il est principalement intéressé par le processus de résolution et non pas seulement par le résultat (S2 J7 Ep. l). 
Finalement, tout cela nous conduit à considérer qu'il y a une stabilisation progressive du contrat des activités 1 à 4 sur trois aspects : la collaboration dans le travail en autonomie, le statut de l'erreur dans la résolution de la tâche et l'importance donnée au fonctionnement du savoir dans la classe au regard du résultat à trouver.

Cette stabilité nous amène aux dernières règles liées aux phases de mise en commun et d'institutionnalisation.

\section{Règles spécifiques aux phases de correction et d'institutionnalisation en classe entière}

Quand les élèves sont en classe entière, le professeur doit demander aux élèves de contribuer au savoir comme on peut le voir dans l'Extrait 11 relatif à l'activité 3, au cours duquel trois élèves (SMA, ELX, THO) appartenant à des groupes différents prennent tour à tour la parole à la suite d'une question du professeur.

\section{Extrait 11}

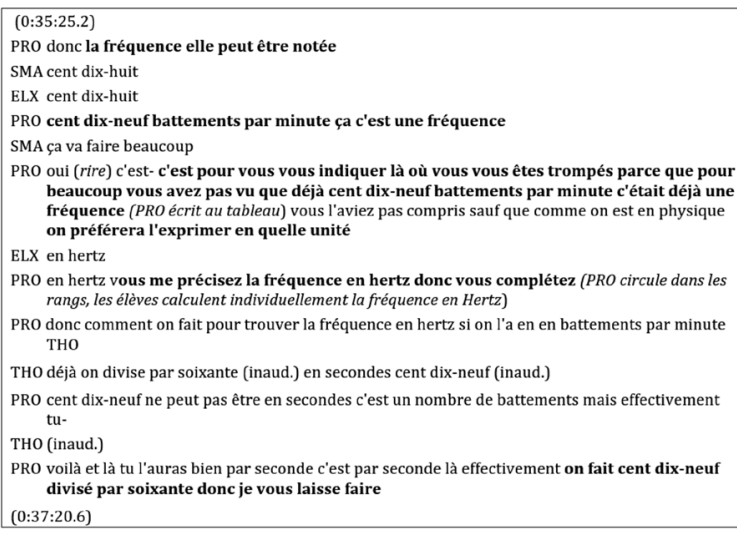

Dans la correction de l'activité 3 en classe entière, les élèves ont à mobiliser la réflexion qu'ils ont menée dans le travail en groupes pour répondre aux questions du professeur ( $\$ 4 \mathrm{~J} 6$ Ep 1 - Activité 3).

Il doit aussi contribuer lui-même de manière dense au savoir comme en témoigne l'Extrait 12 relatif à l'activité 2, dans lequel le professeur est amené à définir la période du passage du tramway dans une station particulière.
Extrait 12

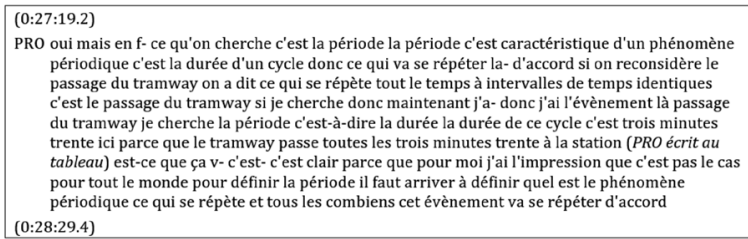

Le professeur contribue lui-même au savoir dans la correction de l'activité réalisée par les élèves en groupes. Ici, il s'agit de déterminer une période à partir des horaires de passage d'un tramway dans l'activité 2. (S2 J3 Ep 11).

Le professeur doit aussi reprendre chacune des questions de l'activité comme on peut le voir dans l'Extrait 11, la détermination par une mesure de la fréquence du haut-parleur faisant l'objet à la fois d'un travail en groupes et de la correction dans le jeu suivant. Il doit aussi expliciter et surtout faire expliciter par les élèves l'ensemble des données empiriques ainsi que les éléments théoriques du savoir ainsi que leur mise en relation. Ainsi dans l'Extrait 13, on voit le professeur dans la correction de l'activité 1 faire expliciter aux élèves la définition de "phénomène périodique » telle qu'existant à ce moment-là dans la classe, puis décrire le mouvement d'un pendule (l'amplitude varie, le mouvement cesse à la fin), et finalement mettre en relation les observations avec la définition pour conclure que ce mouvement n'est pas périodique. 
Extrait 13

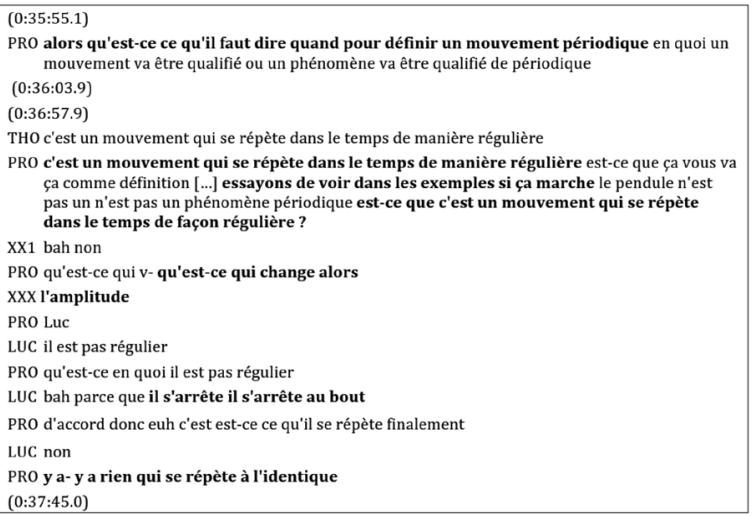

Lors de la correction en classe entière, le professeur fait expliciter les éléments théoriques et les données empiriques ainsi que leur mise en relation (S1 J9 Ep3 - Activité 1).

Enfin, il garantit la pertinence des réponses et les institutionnalise. Dans la suite de la correction de l'activité 1 , le professeur est amené à affiner la notion de mouvement périodique pour finalement arriver à la conclusion qui sera notée sur le cahier de l'élève (cf. Extrait 14).

Extrait 14

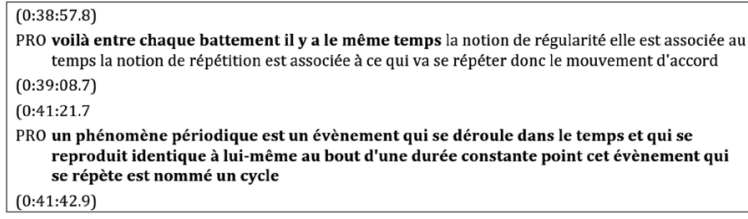
temps la notion de répétition est associée à ce qui va se répéter donc le mouvement d'accord (0:39:08.7)

Les épisodes de niveau N-1 en classe entière concernent la correction de l'activité et l'institutionnalisation des résultats correspondants qui de fait explicite les savoirs à apprendre (S1 J9 Ep 5 Ep. 6).

Finalement, aucun acte du professeur ou des élèves ne vient contredire les règles particulières aux phases de correction et d'institutionnalisation en classe entière élaborées dans l'activité 4 , cellesci sont respectées dans toutes les activités et donc stables tout au long de la séquence.

Après avoir examiné la stabilité de toutes les règles établies dans l'activité 4 à l'échelle mésoscopique (niveau $\mathrm{N}$ ), abordons maintenant la stabilité des règles établies dans l'activité 4 à l'échelle de $1 \mathrm{~h}$ à lh 30 (niveau $\mathrm{N}+1$ ).

\section{Stabilité des composantes établies à l'échelle $\mathrm{N}+1$ dans les autres activités}

Rappelons que, dans l'activité 4, l'identification et la détermination des caractéristiques d'un électrocardiogramme ont été menées, après avoir pris rapidement connaissance de la tâche à réaliser, en construisant en groupe de deux des idées permettant de répondre aux questions posées à partir de ce que les élèves savent déjà, en prenant appui sur ces idées pour corriger et formaliser collectivement les solutions correspondantes, et en institutionnalisant les savoirs nouveaux en jeu dans l'activité. Cette analyse avait permis d'établir les règles du contrat à l'échelle $\mathrm{N}+1$.

Cette structuration de l'action didactique à l'échelle $\mathrm{N}+1$ se retrouve aussi lors du déroulement des trois autres activités, avec toutefois des nuances. Alors que l'activité 2 a exactement cette structure, les activités 1 et 3 présentent quelques écarts.

Dans l'activité 1 , comme nous l'avons déjà évoqué dans une précédente section, la réalisation de la tâche, au lieu d'être entièrement en petits groupes (cf. jeu S1 J7, Tableau 13) est souvent " mixte ». En effet, le professeur et les élèves interagissent par moments en s'adressant à toute la classe : le professeur complète le milieu au fur et à mesure des demandes des élèves notamment en projetant à plusieurs reprises les vidéos des mouvements à étudier et en précisant le sens de certains mots figurant dans l'énoncé de la tâche. En revanche, les jeux S1 J6 (prise de connaissance de l'activité) et S1 J8 et S1 J9 (correction collective et institutionnalisation) sont conformes au pattern apparu dans le déroulement de l'activité 4. Lors de ces deux derniers jeux, les élèves contribuent de manière importante au savoir (dans 4 épisodes sur 6) en décrivant et caractérisant les trois situations observées ; ainsi ces contributions montrent que de nouvelles idées ont été suffisamment construites dans les groupes de deux lors du jeu S1 J7. Ceci nous conduit à considérer que le pattern pour cette activité est semblable à celui de l'activité 4.

Quant à l'activité 3 au cours de laquelle les élèves ont à déterminer la période et la fréquence de vibration de la membrane d'un haut-parleur, comme nous l'avons décrit dans le Tableau 12, son organisation est plus complexe. Après le jeu introductif présentant l'activité, le travail en groupe de deux débute par une phase dans laquelle le professeur règle le maté- 
Tableau 13 : Structuration de l'action didactique relative à l'activité 1 à l'échelle mésoscopique (Niveau N)

\begin{tabular}{|l|l|c|c|}
\hline S1 J6 & $\begin{array}{l}\text { Prendre rapidement connaissance de la tâche « Identifier si un mouvement est pério- } \\
\text { dique à partir de l'observation de trois mouvements » }\end{array}$ & $0: 03: 33$ & Classe entière \\
\hline S1 J7 & $\begin{array}{l}\text { Déterminer en collaborant avec son partenaire, si un mouvement observé est pério- } \\
\text { dique, justifier sa réponse par écrit }\end{array}$ & $\begin{array}{c}0: 08: 49 \\
\text { petit groupe/ } \\
\text { Mixte }\end{array}$ \\
\hline S1 J8 & $\begin{array}{l}\text { Comprendre grâce à la discussion collective la correction des arguments permettant } \\
\text { d'avancer la nature périodique ou non du mouvement observé }\end{array}$ & $0: 04: 50$ & Classe entière \\
\hline S1 J9 & $\begin{array}{l}\text { Comprendre grâce à la discussion collective la définition d'un phénomène périodique, } \\
\text { de la période, du motif. }\end{array}$ & $0: 08: 03$ & Classe entière \\
\hline
\end{tabular}

riel, au cours de laquelle les élèves ne produisent aucune idée nouvelle (jeu S2 J6, durée 05 :19). Par contre, lorsque le matériel est opérationnel, ceux-ci collaborent et avancent dans l'investigation demandée jusqu'à compter un certain nombre de vibrations de la membrane pendant une durée particulière (Jeu S2 J7, durée $36: 40$ ). Après avoir explicité dans une partie de la séance 3 ce qu'on entend par rédaction d'un protocole (en général), le professeur demande à chaque groupe de rédiger à l'extérieur de la classe, le protocole de l'investigation réalisée, en le préparant collectivement et en le rédigeant individuellement. Comme nous l'avons dit précédemment, le professeur insiste d'ailleurs sur la nécessité d'une collaboration $(25: 14) »$. La correction qui suit est collective (Jeux S4 J7 et S4 J9, durée $15: 11$ ), même si pendant un court moment (Jeu S4 J8, durée $1: 51$ ), les élèves ont individuellement à faire le calcul (absent de tous les protocoles) permettant d'exprimer la fréquence en Hertz. Nous considérons toutefois que ce volet individuel dans l'activité est marginal tant au regard de sa durée que du travail effectivement réalisé en groupe de deux ou collectivement en classe entière. De la même manière nous considérons que la phase de mise en marche du matériel est aussi marginale compte-tenu de sa durée et du fait qu'elle n'est pas directement liée à la résolution de la question posée. À ces nuances près, l'action didactique liée à l'activité 3 est donc développée dans la classe à l'instar de toutes les autres

On peut en conséquence donner une description à l'échelle de $1 \mathrm{~h}$ à $1 \mathrm{~h} 30$ de l'action didactique développée dans les quatre activités telle que matérialisée dans le Tableau 14. Comme on peut le voir, la structuration proposée ici est essentiellement liée à la manière dont le savoir est mis en jeu : à cette échelle

Tableau 14 : Structuration de l'action didactique à l'échelle de 1 h à 1 h 30 (niveau N+1) 13

\begin{tabular}{|c|c|c|}
\hline & $\begin{array}{c}\text { Manière dont le savoir est mis en jeu (le professeur orientant généra- } \\
\text { lement les actes liés au savoir, } \\
\text { auxquels l'élève contribue de manière significative) }\end{array}$ & $\begin{array}{c}\text { Enjeu de savoir (fortement prépondérant dans les } \\
\text { actes du professeur et de l'élève) }\end{array}$ \\
\hline \multirow{3}{*}{ 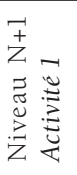 } & Prise de connaissance collective de la tâche donnée & \multirow{3}{*}{$\begin{array}{l}\text { Définition de « phénomène périodique » et de } \\
\text { «période » à partir de l'observation et la compa- } \\
\text { raison de trois mouvements. }\end{array}$} \\
\hline & $\begin{array}{l}\text { Construction de nouvelles idées, pertinentes ou non, en groupe de deux } \\
\text { pour répondre à la tâche en rapportant la démarche }\end{array}$ & \\
\hline & Correction collective et institutionnalisation & \\
\hline \multirow{3}{*}{ 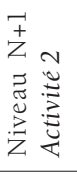 } & Prise de connaissance collective de la tâche donnée & \multirow{3}{*}{$\begin{array}{l}\text { Définition de «fréquence » à partir de deux situa- } \\
\text { tions de la vie quotidienne. }\end{array}$} \\
\hline & $\begin{array}{l}\text { Construction de nouvelles idées pertinentes ou non, en groupe de deux } \\
\text { pour répondre à la tâche en rapportant la démarche }\end{array}$ & \\
\hline & Correction collective et institutionnalisation & \\
\hline \multirow{3}{*}{ 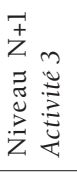 } & Prise de connaissance collective de la tâche donnée & \multirow{3}{*}{$\begin{array}{l}\text { Détermination de la fréquence et la période de } \\
\text { la membrane d'un haut-parleur à partir d'une } \\
\text { investigation expérimentale }\end{array}$} \\
\hline & $\begin{array}{l}\text { Construction de nouvelles idées, pertinentes ou non, en groupe de deux } \\
\text { pour répondre à la tâche en rapportant la démarche }\end{array}$ & \\
\hline & Correction collective et institutionnalisation & \\
\hline \multirow{3}{*}{ 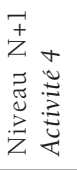 } & Prise de connaissance collective de la tâche donnée & \multirow{3}{*}{$\begin{array}{l}\text { Détermination de fréquences cardiaques à partir } \\
\text { de l'exploitation d'électrocardiogrammes (mesure } \\
\text { de la période) }\end{array}$} \\
\hline & $\begin{array}{l}\text { Construction de nouvelles idées pertinentes ou non, en groupe de deux } \\
\text { pour répondre à la tâche en rapportant la démarche }\end{array}$ & \\
\hline & Correction collective et institutionnalisation & \\
\hline
\end{tabular}


en effet, cette manière est invariante, alors que l'enjeu de savoir change. Cela conduit à dire que les règles dégagées à cette échelle pour le contrat (cf. p. 21) sont valides pour les 4 activités. N'oublions pas enfin la validité à cette échelle des trois règles importantes appliquées à l'ensemble des jeux principaux.

Pour compléter le tableau donné à cette échelle de temps, il faut signaler que certains jeux de la séquence sont extérieurs aux quatre activités proposées et ne s'insèrent pas dans cette structuration. Si certains concernent conjoncturellement la correction d'un exercice de la séquence précédente, d'autres viennent en appui des activités proposées sur les phénomènes périodiques (voir Tableau 15). Ainsi au cours des jeux S3 J3, S3 J4 et S3 J5, le professeur explique comment structurer (en général) un protocole, et exemplifie régulièrement ses propos à partir d'éléments de l'activité 3 fournis par les élèves. Les jeux S4 J3 et S4 J4 sont dédiés quant à eux à la correction collective en classe entière d'exercices préparés en dehors de la classe en relation directe avec le thème traité : identification de la grandeur représentée dans un électrocardiogramme et de sa périodicité et calcul en notation scientifique de la période à partir de la fréquence en se basant sur la définition des deux grandeurs. Si lors de la présentation de ce qu'est un protocole on est plutôt dans un cours dialogué, dans la correction des exercices, il s'agit de développer en classe entière un savoir déjà introduit avec une contribution importante des élèves (mise en commun à partir du travail individuel) et à l'initiative du professeur. Les composantes du contrat dégagées dans les phases collectives des activités valent donc aussi pour la correction des exercices.

\section{Conclusion}

Au cours de cette partie de l'étude dans laquelle nous avons souhaité vérifier la validité des règles du contrat établies sur l'activité 4, deux nouvelles règles sont apparues, l'une liée à la constitution d'un milieu adéquat pour rendre possible le travail en autonomie, l'autre relative à l'importance donnée au fonctionnement du savoir dans la classe par rapport au résultat à trouver :

- le professeur doit rendre disponible aux élèves un milieu adéquat à la réalisation en autonomie de la tâche ;

- résoudre une tâche en classe de physique c'est avant tout être capable de construire une démarche en utilisant des savoirs déjà enseignés et de l'expliquer, le résultat restant important mais pas suffisant.

Ces règles n'ont pas été perçues dans l'activité 4 car stabilisées au cours des premières activités, et devenues ensuite implicites dans la classe. D'autres règles se sont stabilisées au cours des premières activités : la prépondérance des actes liés au savoir lors du travail en groupes, le travail en collaboration dans les groupes, le statut de l'erreur dans la résolution de la tâche ou encore la nécessité d'écrire sur son cahier plus que son résultat.

Tableau 15 : l'activité 3 est interrompue au cours de la séance S3 et au début de la séance S4 pour donner lieu à des activités collectives venant en appui des activités 3 et 4

\begin{tabular}{|c|c|c|}
\hline \multirow{4}{*}{ 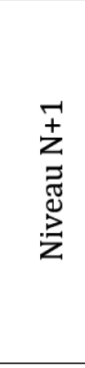 } & Prise de connaissance collective de la tâche donnée & \multirow{2}{*}{$\begin{array}{c}\text { Détermination de la fréquence et la } \\
\text { période de la membrane d'un haut- } \\
\text { parleur à partir d'une investigation } \\
\quad \text { expérimentale (Activité 3) }\end{array}$} \\
\hline & $\begin{array}{l}\text { Construction de nouvelles idées en groupe de deux pour } \\
\text { répondre à la tâche en rapportant la démarche }\end{array}$ & \\
\hline & \multicolumn{2}{|c|}{ Enseignement inséré au miliev de I’activité 3 (S3 et début de S4) } \\
\hline & Correction collective et institutionnalisation & $\begin{array}{l}\text { Détermination de la fréquence et la } \\
\text { période de la membrane d'un haut- } \\
\text { parleur à partir d'une investigation } \\
\quad \text { expérimentale (Activité 3) }\end{array}$ \\
\hline$\sum_{z}^{\bar{z}}$ & $\begin{array}{l}\text { Développement d'éléments en appui des activités } 3 \text { et } 4: \\
\text { Principes pour la rédaction d'un protocole (jeux S3 J3, S3 J4 } \\
\text { et S3 J5) - Correction de deux exercices opérationnalisant } \\
\text { des savoirs déjà introduits (jeux S4 J3 et S4 J4) }\end{array}$ & $\begin{array}{l}\text { Calcul de la période à partir d'une } \\
\text { fréquence - Détermination de la } \\
\text { grandeur représentée sur un ECG et } \\
\text { de sa périodicité }\end{array}$ \\
\hline
\end{tabular}


Enfin, rappelons les trois règles importantes communes à l'ensemble des jeux principaux et de ce fait, valides aux échelles supérieures : les actes du professeur et des élèves sont essentiellement centrés sur le savoir, les élèves y prennent une part significative, le déroulement des actes est orienté par l'enseignant qui en garde l'initiative. Parce qu'elle concerne le fonctionnement du savoir dans la classe, la règle relative à la nécessaire explicitation de la démarche suivie pour aboutir au résultat peut être aussi entendue comme " règle importante », même si elle n'est pas toujours apparente.

Lors de la quatrième et dernière phase de l'investigation, il y a à construire les règles du contrat aux échelles supérieures de temps, ce que propose la section suivante.

Figure 11 : les sections suivantes décrivent les résultats de la phase 4 de la méthode d'investigation

Ph 1 : Recueillir, transcrire et structurer les données

Ph 2: Analyser l'activité 4 aux échelles $\mathrm{N}-2$ à $\mathrm{N}+1$ formaliser les règles correspondantes du contrat

Ph 3 : Analyser les activités 1,2 et 3 aux échelles $\mathrm{N}-2$ à $\mathrm{N}+1$, vérifier et compléter les règles établies sur l'activité 4

Ph 4 : Analyser la séquence aux échelles $\mathrm{N}+2$ et $\mathrm{N}+3$, formaliser les règles correspondantes du contrat

\section{COMPOSANTES DU CONTRAT AU NIVEAU N+2, ET $\mathbf{N}+3$}

\section{Composantes au niveau $\mathrm{N}+2$ (échelle de 2 à $3 \mathrm{~h}$ )}

Si dans la structuration précédente (Tableau 14), on centre son attention sur le savoir en jeu, on voit apparaître une organisation particulière de l'action didactique à l'échelle de 2 à 3 h environ, qui définit pour nous le niveau N+2 (cf. Tableau 16). Ainsi, à ce niveau, il apparaît que, dans la première partie de la séquence, il s'agit d'abord de construire des descriptions adéquates des événements associés aux phénomènes périodiques pour ensuite établir les savoirs relatifs aux phénomènes périodiques à un niveau théorique à partir des relations entre monde empirique et monde théorique, alors que dans la seconde partie, il s'agit d'opérationnaliser ces savoirs sur deux situations expérimentales, et donc de se les approprier plus en profondeur. Cela implique d'ailleurs un degré de maîtrise minimal à l'entrée de cette seconde partie. Ce point de vue prend en compte le but dans lequel on manipule les savoirs, qui organise l'action didactique à l'échelle de trois heures environ : les définir pour être en mesure de les utiliser même d'une manière incertaine dans des situations nouvelles, ou les rendre plus opérationnels en travaillant sur de nouvelles situations et donc les maîtriser plus en profondeur. Tout ceci se fait rappelons-le, le plus souvent sous l'orientation du professeur avec la contribution significative des élèves qui peuvent proposer des idées plus ou moins correctes et ont à expliciter leur démarche.

Le sens donné au niveau $N+2$ de l'analyse de l'action didactique effectuée au niveau $\mathrm{N}+1$ ajoute d'autres composantes au contrat :

- Lélève doit contribuer à partir de situations matérielles à la formalisation des savoirs (élaboration de définitions relatives à l'espace théorique en relation avec l'espace empirique) dans la première partie de la séquence et étudier les savoirs plus en profondeur dans la seconde en les mobilisant sur des situations physiques.

- L'élève doit comprendre les savoirs d'une manière minimale avant d'entrer dans la seconde partie de la séquence, et doit les comprendre de manière plus approfondie en les mettant en œuvre dans quelques situations en fin de seconde partie.

- Le professeur doit garantir à travers son action une compréhension minimale des savoirs par les élèves à la fin de la première partie de la séquence, et une compréhension plus approfondie en fin de seconde partie.

On ne peut pas dire si ces règles relèvent vraiment de la dimension durable du contrat, dans la mesure où notre étude se limite à une seule séquence. Il serait en effet nécessaire d'observer plusieurs séquences pour établir ou non leur récurrence.

\section{Composantes au niveau N+3 (échelle de $5-6 \mathrm{~h}$ )}

En interprétant au niveau N+3 (niveau macroscopique) l'analyse de l'action didactique effectuée au niveau $\mathrm{N}+2$, (cf. Tableau 17), on peut compléter les composantes du contrat par la règle suivante :

- Lélève doit identifier et caractériser un phénomène périodique dans différents contextes.

Cette règle qui met en jeu les analyses au niveau $\mathrm{N}+1, \mathrm{~N}+2$ et $\mathrm{N}+3$, rend aussi compte de ce qu'est ici 
Tableau 16: ${ }^{14}$ Structuration de l'action didactique à l'échelle de deux ou trois heures (Niveau N+2 - Tableau de droite)

\begin{tabular}{|c|c|c|c|c|c|}
\hline \multirow{3}{*}{ 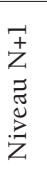 } & Prise Con. & \multirow{3}{*}{$\begin{array}{l}\text { Définition de "phénomène périodique " et } \\
\text { de "période " à partir de l'observation et la } \\
\text { comparaison de trois mouvements. }\end{array}$} & \multirow{13}{*}{ 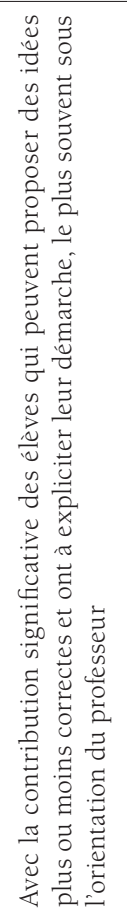 } & \multirow{6}{*}{ 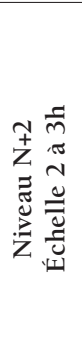 } & \multirow{6}{*}{$\begin{array}{l}\text { Établissement des savoirs } \\
\text { relatifs aux phénomènes pério- } \\
\text { diques aux niveaux empirique } \\
\text { et théorique en construisant } \\
\text { des relations entre théorie et } \\
\text { monde matériel }\end{array}$} \\
\hline & Rés Coll. & & & & \\
\hline & Corr. & & & & \\
\hline \multirow{3}{*}{ 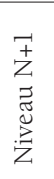 } & Prise Con. & \multirow{3}{*}{$\begin{array}{c}\text { Définition de "fréquence " à partir de deux } \\
\text { situations de la vie quotidienne. }\end{array}$} & & & \\
\hline & Rés Coll. & & & & \\
\hline & Corr. & & & & \\
\hline \multirow{3}{*}{ 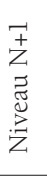 } & Prise Con. & \multirow{3}{*}{$\begin{array}{c}\text { Détermination de la fréquence de la membrane } \\
\text { d'un haut-parleur à partir d'une investigation } \\
\text { expérimentale }\end{array}$} & & \multirow{7}{*}{ 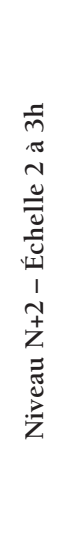 } & \multirow{7}{*}{$\begin{array}{l}\text { Opérationnalisation des } \\
\text { savoirs relatifs aux phéno- } \\
\text { mènes périodiques en établis- } \\
\text { sant des relations entre théorie } \\
\text { et situations physiques }\end{array}$} \\
\hline & Rés Coll. & & & & \\
\hline & Corr. & & & & \\
\hline 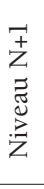 & & $\begin{array}{c}\text { Définition de ce qu'est un protocole. Calcul de } \\
\text { la période à partir de la fréquence, identifica- } \\
\text { tion de la grandeur représentée sur un ECG et } \\
\text { de sa périodicité (exercices) }\end{array}$ & & & \\
\hline \multirow{3}{*}{ 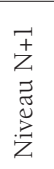 } & Prise Con. & \multirow{3}{*}{$\begin{array}{c}\text { Détermination de fréquences cardiaques à } \\
\text { partir de l'exploitation d'électrocardiogrammes } \\
\text { (mesure de la période) }\end{array}$} & & & \\
\hline & Rés Coll. & & & & \\
\hline & Corr. & & & & \\
\hline
\end{tabular}

Prise Con. : prise de connaissance de la tâche; Rés Coll. : résolution collaborative de la tâche; Corr. : correction de la tâche et institutionnalisation.

« savoir », d'autant plus que les élèves sont ensuite évalués. On a donc une règle supplémentaire qui caractérise le niveau N+3 (cf. Tableau 17) :

- Savoir, c'est être en mesure en fin d'enseignement de définir les savoirs correspondants et aussi de les utiliser en contexte en expli- citant le cheminement suivi et en particulier en mettant en relation les éléments théoriques avec le monde matériel.

Le professeur doit en effet garantir en fin d'enseignement que l'élève maîtrise de manière suffisante la reconnaissance et la caractérisation d'un phénomène

Tableau 17 : Structuration de l'action didactique à l'échelle de 5 à 6 heures (Niveau N+3 - Tableau de droite)

\begin{tabular}{|c|c|}
\hline 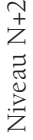 & $\begin{array}{l}\text { Établir les savoirs relatifs aux phénomènes pério- } \\
\text { diques à un niveau théorique à partir de relations } \\
\text { entre théorie et monde physique }\end{array}$ \\
\hline 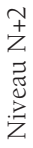 & $\begin{array}{l}\text { Opérationnaliser les savoirs relatifs aux phénomènes } \\
\text { périodiques en établissant des relations entre théorie } \\
\text { et situations physiques }\end{array}$ \\
\hline
\end{tabular}

\begin{tabular}{|c|c|}
\hline 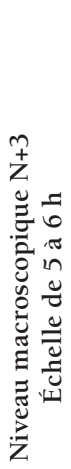 & $\begin{array}{l}\text { I dentifier et caractériser un } \\
\text { phénomène périodique dans différents } \\
\text { contextes, à partir de la résolution de tâches } \\
\text { mettant en jeu la construction collaborative } \\
\text { d'idées nouvelles pertinentes ou non basées } \\
\text { sur ce que l'on sait, en explicitant la démarche } \\
\text { élaborée, et sous le pilotage du professeur. }\end{array}$ \\
\hline & Évaluation \\
\hline
\end{tabular}


périodique pour qu'il soit en mesure de faire face à l'évaluation dont il fait l'objet.

\section{DISCUSSION}

Cet ensemble conséquent de résultats étant établi, il reste à les discuter, d'abord sur le plan méthodologique, en revenant sur les échelles mobilisées ainsi que sur d'autres potentialités de la méthode que nous avons développée, puis sur le plan du contrat décrit.

\section{Aspects méthodologiques}

\section{Échelles mobilisées}

Notre étude a mobilisé six échelles temporelles rappelées dans la Figure 12. description du contrat.Par ailleurs, pour identifier les règles constitutives de la dimension durable du contrat, nous avons recherché des régularités dans les descriptions de l'action didactique à différentes échelles. Cette stratégie a fonctionné de manière satisfaisante jusqu'au niveau $\mathrm{N}+1$ (échelle de $1 \mathrm{~h}$ à $1 \mathrm{~h} 30$ ), défini à partir de régularités concernant le niveau $\mathrm{N}$ (échelle de 10 à 30 minutes). Les régularités observées au niveau $\mathrm{N}+1$ qui ont permis de structurer et caractériser le niveau $\mathrm{N}+2$ ont un caractère plus hypothétique car leur récurrence n'est pas réellement établie, il aurait fallu pour cela observer et analyser au minimum deux séquences et non pas une seule. Quant aux caractéristiques qui émergent du niveau $\mathrm{N}+2$ (échelle de 2-3 h) pour définir le niveau $\mathrm{N}+3$ (échelle de 5-6 heures), elles ont été observées à une seule reprise compte tenu des temporalités en jeu. En conséquence, si les règles établies aux échelles de temps élevées ont une validité pour la

Figure 12 : les six échelles mobilisées dans cette étude

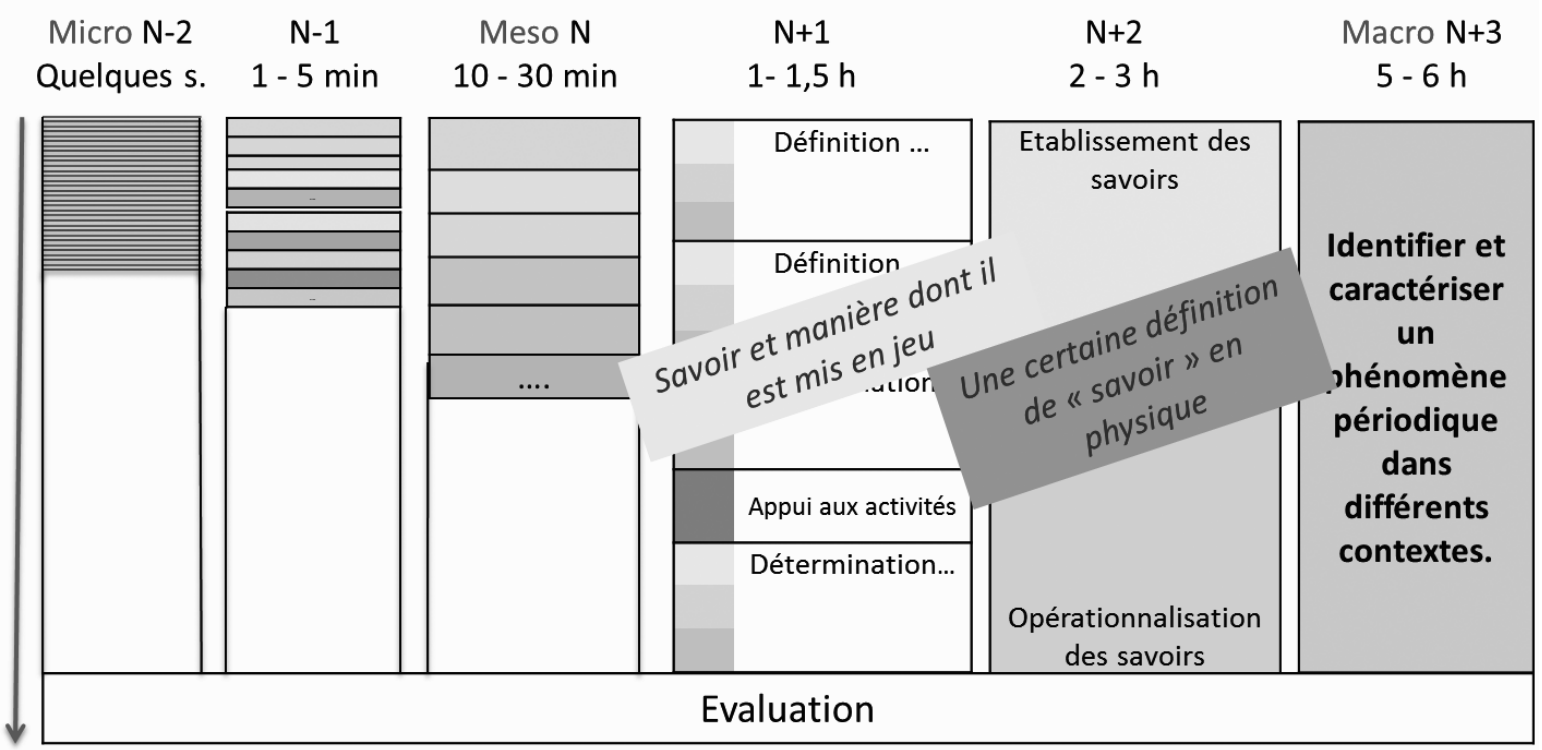

Temps

On peut tout d'abord souligner que chacune de ces six échelles de temps est nécessaire pour décrire le contrat didactique. En effet, seule l'échelle «1-5 minutes " n'a pas été directement exploitée pour formaliser des règles, car l'homogénéité des épisodes $\mathrm{N}-1$ que nous avons montrée a permis de les produire à l'échelle mésoscopique. Ainsi, en omettant une échelle, on perd une partie de la séquence observée, il reste à montrer leur récurrence pour qu'elles relèvent pleinement de la dimension « durable » du contrat, en observant la classe sur un empan temporel d'au moins deux ou trois séquences.

Nos analyses nous ont amenés au cours de cette étude à rajouter deux échelles nouvelles par rapport aux études précédentes, 1 à $1 \mathrm{~h} 30$ et 2 à $3 \mathrm{~h}$. La première a été définie à partir du savoir concerné 
et de la manière dont il est mis en jeu, centrée sur un travail en autonomie par groupe de deux et d'une correction collective avec institutionnalisation. La seconde quant à elle est reliée à ce que veut dire "savoir » dans cette séquence, c'est-àdire être capable à la fois de définir des concepts et les relations qui les unissent, et d'exploiter ces éléments dans des contextes particuliers. Toutefois, si ces échelles et les éléments sur lesquels elles sont fondées sont valides sur cette séquence basée sur un enseignement par activités, la question de leur validité sur d'autres formats d'enseignement et d'autres séquences est ouverte, et donc en même temps celle de leur généralisation, qui ira de pair avec l'établissement d'outils théoriques associés.

Enfin, nous avons peu attiré l'attention dans la présentation des résultats sur une autre échelle que nous avons mobilisée, celle qui est exploitée pour isoler les facettes et surtout les tâches épistémiques. Nous avons simplement dit que facettes et tâches pouvaient s'étaler sur plusieurs actes définissant par-là implicitement une échelle intermédiaire entre les niveaux $\mathrm{N}-2$ et $\mathrm{N}-1$. Cela montre bien que les échelles mobilisées dans les études n'ont rien d'absolu mais sont bien en lien avec les théories mobilisées, et les questions de recherche correspondantes.

\section{Potentialités de la méthode développée}

Nous avons débuté par la construction des règles relatives à l'activité 4 à partir des analyses des actes du professeur et des élèves au niveau microscopique selon les trois dimensions pragmatiques, interactionnelles et représentationnelles, analyses que nous avons interprétées aux échelles N-1 et N. Dans cette interprétation, chaque règle explicite des actions du professeur et des élèves intégrant les trois dimensions sans les distinguer. Ainsi à partir des actes, de leur importance respective, nous avons inféré des règles au niveau N. Dans cette démarche, nous avons été guidés par les concepts de contrat et milieu qui mettent en avant la relation professeur - élève savoir et plus précisément les stratégies pour jouer au jeu associées à un enjeu de savoir. Ainsi, toutes les règles, à l'exclusion de l'une d'entre elles, rendent compte de la stratégie du professeur et/ou de l'élève pendant le jeu. La règle qui fait exception " les élèves doivent s'approprier le réseau conceptuel relatif à "phénomène périodique" " est appa- rue comme nécessaire pour «nourrir » les stratégies qui ne peuvent tourner à vide, elle relève de l'enjeu d'apprentissage alors que toutes les autres sont relatives à des actions du professeur et des élèves dans la classe. Ce sont ces dernières que nous avons qualifiées de «durables » dans la mesure où elles ne sont pas spécifiques de l'élément de savoir en jeu. Leur durée est au moins celle de la séquence, mais elles peuvent ensuite évoluer, elles peuvent aussi laisser la place à certains moments à des règles très différentes, comme on peut le voir par exemple quand le professeur explique lui-même et seul, en interrompant l'activité 3, comment un protocole doit être rédigé dans la classe de physique. Leur caractère " durable » est donc relatif.

Nos analyses ont donc permis de spécifier un certain nombre de règles relevant de cette dimension « durable» du contrat, de montrer aussi que certaines avaient été établies rapidement, et que d'autres avaient évolué préalablement à leur stabilisation. En ce sens, l'étude des transactions au niveau microscopique pour spécifier des composantes du contrat a porté ses fruits, et offre un progrès par rapport à des analyses conduites précédemment. Celles-ci étaient en effet souvent basées sur l'expertise du chercheur qui lui permettait notamment d'identifier des ruptures de contrat et en conséquence de caractériser une de ses composantes. Dans cette étude, les composantes sont recherchées de manière plus systématique et sont fondées empiriquement sur plusieurs critères empreints d'objectivité, ou au moins de critères qui peuvent faire l'objet d'une référence objective commune pour en débattre (même si bien sûr on ne peut laisser penser pour autant qu'elles sont totalement exemptes de subjectivité).

Au-delà de l'usage développé dans cette étude, d'autres exploitations de la méthode utilisée sont possibles, dans des contextes différents, ou encore avec d'autres objectifs, comme la recherche de contrats différentiels ou encore la description des règles du contrat, spécifiques du savoir en jeu.

En effet, les diverses études empiriques que nous avons développées au niveau N-2 (voir par exemple Tiberghien et Venturini, 2015) nous ont permis de constituer un catalogue non exhaustif de catégories pour les différentes dimensions, pragmatiques, interactionnelles, et représentationnelles nettement plus vaste que celui utilisé ici. Mais celles que nous exploitons in fine dans une analyse particulière sont liées à la séquence observée et à la question de recherche. 
Ainsi, dans cette étude, nous n'avons pas jugé utile de raffiner par exemple des catégories concernant la gestion de la classe et de la communication parce que les actes correspondants sont minoritaires. Mais il est possible si nécessaire de distinguer les actes relevant de la gestion ordinaire de la communication des actes associés à la gestion « disciplinaire», voire de raffiner encore l'une ou l'autre d'entre elles. De la même manière, nous n'avons pas raffiné par exemple la catégorie « questions » apparaissant dans la dimension interactionnelle en questions ouvertes ou fermées, questions de relance etc., parce que la quasi-totalité des questions apparaissant dans cette séquence sont fermées, ou encore nous n'avons pas mobilisé dans les tâches épistémiques des tâches d'évaluation/comparaison ou d'argumentation car celles-ci sont inexistantes ou marginales. Aussi si, les trois dimensions sont toujours présentes dans notre grille d'analyse au niveau microscopique, les catégories qu'elles comportent, leur degré de raffinement varient selon le contexte de la recherche. Cette " malléabilité » nous paraît être une caractéristique à porter au crédit de la méthode développée, celle-ci étant en conséquence utilisable dans des contextes très différents.

Par ailleurs, l'analyse systématique qui est développée permet aussi de mettre à jour des contrats différentiels. Nous n'avons pas exploité cette possibilité dans cette étude parce que les données s'y prêtent peu, nous avons au contraire montré une homogénéité dans les interventions, notamment dans les différents groupes de deux. Mais dans le cas d'une classe plus hétérogène, il est possible de faire apparaître des différences dans le contrat avec différents élèves.

Enfin, en exploitant la dimension représentationnelle plus finement que nous ne l'avons fait ici, il est possible de rendre compte de l'évolution de la chronogenèse et de l'apparition progressive des règles du contrat liées à l'enjeu de savoir ou leur rupture.

Tout cela ne veut pas dire pour autant que la méthode développée ne présente pas certaines limites. Ainsi par exemple, une des règles établies au niveau N+1 fait état de la nécessité pour les élèves de s'engager dans la résolution de la tâche donnée dans l'activité, en collaboration avec leurs partenaires. Mais par comparaison avec une étude similaire menée dans une autre classe, nous avons des indices à d'autres échelles (par exemple présence régulière en cours, travaux à la maison réalisés...) pour avancer que l'engagement des élèves dépasse largement la résolution des tâches données. Ainsi, la méthode développée ne garantit pas l'exhaustivité dans les règles qu'elle permet de construire.

\section{Particularités du contrat dans cette séquence}

Remarquons tout d'abord que dans le cas observé, certaines règles relatives aux niveaux $\mathrm{N}-2$ à $\mathrm{N}+1$ sont spécifiques de l'organisation de la classe, par groupes de deux ou en classe entière. D'autres qui concernent les niveaux $\mathrm{N}$ à $\mathrm{N}+3$ sont valides quelle que soit cette organisation. Par ailleurs, la plupart des composantes de ce contrat sont soit déjà mises en place (pour les phases de correction par exemple) soit établies rapidement. On peut penser que les habitudes de travail prises lors des travaux pratiques classiques dans lesquels le travail se déroule aussi par deux ont servi de tremplin pour la compréhension des attentes du professeur par les élèves. D'ailleurs, celui-ci s'appuie sur ces similarités : ainsi il annonce min 18 lors de la première séance «vous avez le droit de travailler à deux comme en TP » ou encore min 25 «vous travaillez à deux comme en TP ». La régularité observée dans l'organisation et le déroulement du travail tout au long de la séquence contribue aussi certainement à la stabilisation rapide du contrat et offre aux élèves un environnement dans lequel ils ont pu rapidement construire des repères.

Il est aussi intéressant de remarquer que les deux règles que nous avons rajoutées à la suite des analyses des trois premières activités correspondent à des actions qui ont une portée beaucoup plus générale. La première (le professeur doit rendre disponible aux élèves un milieu adéquat à la réalisation en autonomie de la tâche) renvoie à la nécessité pour le professeur de préparer le milieu avant la situation d'enseignement. Il lui est en effet difficile d'y déroger, même si conjoncturellement il peut pallier l'absence de quelques éléments du milieu en les apportant lui-même lorsqu'il est en classe entière. La seconde ( "savoir" quelque chose en classe de physique c'est avant tout être capable de construire une démarche en utilisant des savoirs déjà enseignés et de l'expliquer, le résultat restant important mais pas suffisant ») va bien au-delà de la classe de physique, elle oriente la façon de travailler y compris en dehors de l'école. 
Enfin, le contrat en cours dans la classe lors de cette séquence laisse un espace important au travail des élèves, et rend compte d'une démarche d'apprentissage progressive au cours de laquelle ils ont à mobiliser à la fois ce qu'ils savaient déjà et ce qu'ils sont en train d'apprendre dans un contexte où l'erreur est toujours possible. Mais il fait aussi une place importante au professeur qui oriente quasiment en permanence l'action didactique, tout en laissant souvent les élèves reprendre la main une fois qu'il l'a orientée.

\section{CONCLUSION ET PERSPECTIVES}

Pour conclure, nous souhaitons mettre ou remettre l'accent sur certains points, certains d'entre eux ayant été abordés dans la discussion.

L'action didactique est une action humaine complexe se déroulant à différentes temporalités. Notre étude montre qu'il est possible de rendre compte, au moins partiellement, de cette complexité, pour peu que les échelles d'analyse prises en considération soient pertinentes pour les phénomènes investigués.

L'étude confirme aussi la pertinence des outils théoriques proposés, ancrés dans l'action, et cohérents au niveau pragmatique. Ces outils que nous avons construits pour le niveau microscopique ont en effet été déjà testés pour analyser plusieurs séances différentes (Tiberghien et Venturini, 2015). Ils témoignent ici de l'intérêt de leur noyau (dimensions pragmatiques, interactionnelles et représentationnelles) et de leur plasticité dans la mesure où les descripteurs correspondant peuvent s'adapter à des questions et un contexte différents. Leur combinaison avec les outils de la TACD (qui ne sont pas opérationnels au niveau microscopique) permet, lorsque l'on utilise cette dernière à l'échelle mésoscopique, de produire des assertions beaucoup mieux fondées. L'ensemble, associé au type de données recueillies grâce à la vidéo, nous paraît donc bien adapté à l'investigation pragmatique au cœur de la méthode présentée dans cet article.

Celle-ci ouvre aussi de nouvelles perspectives, relatives à son opérationnalité et à son évolution, à la production de nouveaux savoirs sur les pratiques, et à son intérêt pour la formation des enseignants.

Ainsi, on peut supposer que cette méthode permette de documenter la partie du contrat didac- tique spécifique du savoir en jeu que nous n'avons pas explorée dans cette étude, mais il reste à savoir si des adaptations sont nécessaires pour cela. La méthode permet aussi de mettre à jour et de caractériser des contrats différentiels ou encore de comparer les contrats rendant compte des pratiques d'étude et d'enseignement dans deux contextes différents. Ces deux perspectives que nous avons testées doivent permettre d'enrichir nos connaissances sur le fonctionnement des systèmes didactiques, même si pour cela, il reste à adapter les descripteurs de la dimension représentationnelle des actes à d'autres disciplines. Les descripteurs que nous avons utilisés sont en effet spécifiques de la physique.

La question des échelles « longues ", de la pertinence ou non de celles que nous avons proposées issues d'une analyse empirique dans un contexte particulier, la construction des outils théoriques correspondants pour structurer les séquences à ces échelles sont autant de questions en suspens auxquelles l'analyse de différents " formats » d'enseignement sur des durées égales au moins à la séquence pourrait commencer à répondre.

Sur un plan très différent, ce type d'analyse très fine pourrait aussi servir à déterminer des indicateurs lexicaux ou grammaticaux correspondant à des caractéristiques particulières d'actes. Ces éléments permettraient ultérieurement une certaine automatisation des analyses, notamment pour des corpus conséquents. L'instrumentation des recherches de ce type, très coûteuses en temps nous paraît en effet devoir être une perspective à toujours conserver en arrière-plan par les chercheurs pour peu à peu développer des stratégies d'analyse plus économiques.

Enfin, l'option que nous avons prise, grâce à notre orientation pragmatique, de formuler le contrat en termes de règles d'action nous paraît une perspective intéressante pour la formation. En effet, si l'on en juge par la première réaction des professeurs et professeures concernés par le projet qui a donné lieu à cette étude auxquels ces règles ont été présentées, elles sont susceptibles d'être reconnues comme composante d'une pratique professionnelle. Cette formalisation de l'action explicitant des éléments de la pratique, constitue donc vraisemblablement un moyen privilégié de transmettre des pratiques aux professionnels. 


\section{NOTES}

1. Nous avons emprunté cette caractérisation à l'un des relecteurs de l'article.

2. Le projet ASSIST-ME étudie la mise en œuvre combinée de l'évaluation sommative et formative dans un enseignement de sciences expérimentales (Physique, Chimie et Sciences de la vie et de la Terre), de technologie et de mathématiques fondé sur les démarches d'investigation. Ce projet ( $n^{\circ}$ o 321428 ) s'inscrit dans le programme Science Avec et Pour la Société (SwafS) de la Commission Européenne.

3. Voir annexe 1 l'énoncé de la tâche à réaliser dans l'activité 4

4. Nous n'avons pas distingué les deux configurations dans nos analyses, nous avons considéré qu'il s'agissait à chaque fois d'un groupe « classe entière ».

5. Cette formulation "épisode de niveau N-1» sera souvent abrégée dans le texte en « épisode N-1 » pour en alléger la lecture.

6. Classe entière s'oppose ici à travail en groupe de deux. Selon les séances la classe entière comporte 18 élèves ou 36 selon que la classe est dédoublée ou non. Par ailleurs, pour les distinguer, nous avons désigné les épisodes en groupes de deux Ep a, Ep b etc. et les épisodes en classe entière Ep 1, Ep 2 etc.

7. Les jeux sont désignés par le numéro de la séance et le numéro du jeu dans la séance. Le $4^{\mathrm{e}}$ jeu de la séance 1 est ainsi désigné Jeu S1 J4. Chaque séance commence donc par le jeu dénommé Si J1. Si l'activité 4 commence au jeu S4 J8, c'est que la séance 4 comporte d'autres jeux auparavant, qui ne sont pas liés à cette activité.

8. Les concepts scientifiques sont essentiellement relationnels. Les concepts de phénomènes périodiques, cycle, période, fréquence sont liés les uns aux autres et constituent le « réseau conceptuel » en jeu dans la séance.

9. Les interprétations sont majoritaires dans le jeu 9 et les descriptions majoritaires dans le jeu 10. Un test statistique confirme d'ailleurs que les tâches épistémiques mises en jeu dans les deux jeux sont différentes (rejet de l'hypothèse nulle : $(0.05<\mathrm{p}<0.02$, chi $2=6.5$ pour $\mathrm{dl}=2)$

10. Les règles du contrat déjà établies et dont il s'agit de vérifier la stabilité sont mentionnées dans la suite du texte en italique pour les différencier du texte courant.

11. Pour ce faire, les élèves peuvent compter le nombre de vibrations de la membrane du haut-parleur pendant un temps déterminé. La fréquence en Hertz s'obtient en calculant alors le nombre de vibrations pendant une seconde et la période en calculant la durée séparant deux vibrations.
12. Les jeux d'introduction des activités étant extrêmement brefs, on peut considérer que ces trois règles sont communes à l'ensemble des jeux. Chaque échelle supérieure rassemblant un certain nombre de jeux dans lesquels ces trois règles s'appliquent, celles-ci caractérisent aussi le déroulement de l'action didactique à cette échelle.

13. Le tableau 14 dans le tapuscrit avait des niveaux de gris différents sur la colonne de gauche. Je les ai remis.

14. Même remarque sur l'absence de niveaux de gris (les mêmes que dans le tableau précédent, puisque ce sont les mêmes phases). Je remets des niveaux de gris ici.

\section{RÉFÉRENCES}

Brousseau, G. (1997). Theory of Didactical Situations in Mathematics. In N. Balachef, M. Cooper, R. Sutherland, V. Warfield, Eds., Collected Papers. Dordrecht : Kluwer. Bourdieu, P. (1992). Language and Symbolic Power. Cambridge : Polity Press.

Charaudeau, P. (2004). Comment le langage se noue à l'action dans un modèle socio-communicationnel du discours. De l'action au pouvoir. Cahiers de linguistique française, Les modèles du discours face au concept d'action, Actes du 9e colloque de Pragmatique de Genève et colloque Charles Bally, université de Genève, Genève, 26, [en ligne: http://www.patrick-charaudeau.com/ Comment-le-langage-se-noue-a-l,90.html].

Chinn, C. (2006). Classroom Interaction in Science : Teacher questioning and feedback to students' responses. International Journal of Science Education, 28(11), 1315-1346.

Cobb, P., Stephan, M., McClain, K., \& Gravemeijer, K. (2001). Participating in Classroom Mathematical Practices. Journal of the Learning Sciences, 10, 113-163.

Galili, I., \& Hazan, A. (2000). The influence of an historically oriented course on students' content knowledge in optics evaluated by means of facets-schemes analysis. American Journal of Physics, 68 (Supplement)(7), S3-S15.

Kerbrat-Orecchioni, C. (1995). Où en sont les actes de langage?. L'Information Grammaticale, 66, 5-12.

Lemke, J. L. (2000). Across the Scales of Time : Artifacts, Activities, and Meanings in Ecosocial Systems. Mind, culture, and activity, 7(4), 273-290.

Ligozat, F., Lundqvist, E., \& Amade-Escot, C. (2018). Analysing the continuity of teaching and learning in classroom actions: When the joint action framework in didactics meets the pragmatist approach to classroom discourses. European Educational Research Journal, 17(1), 147-169.

McClain, K., \& Cobb, P. (2001). An Analysis of Development of Sociomathematical Norms in One First-Grade Classroom. Journal for Research in Mathematics Education, 32(3), 236-266. 
Mead, G.H. (1938). The Philosophy of the Act. Chicago : The University Chicago Press.

Minstrell, J. (1992). Facets of students' knowledge and relevant instruction. In R. Duit, F. Goldberg \& H. Niedderer (Eds.), Research in physics learning : Theoretical issues and empirical studies (p. 110-128). Kiel : IPN.

Mortimer, E., \& Scott, P. (2000). Analysing discourse in the science classroom. In R. Millar, J. Leach \& J. Osborne (Eds.), Improving science education : the contribution of research (p. 126-142). Buckingham UK : Open University Press.

Nespor, J. (2004). Educational scale-making. Pedagogy, Culture and Society, 12(3), 309-326.Ohlsson, S. (1996). Learning to do and learning to understand: A lesson and a challenge for cognitive modeling. In P. Reiman $\&$ H. Spada (Eds.), Learning in Humans and Machine (p. 37-62). Oxford : Pergamon Elsevier Science.

Searle, J. R. (1969/1972). Les actes de langage. Paris : Hermann

Searle, J. R., \& Vanderveken, D. (1985). Foundations of illocutionary logic. Cambridge : Cambridge University Press.

Sensevy, G., Mercier, A. (2007). Des catégories pour décrire et comprendre l'action didactique. In G. Sensery et A. Mercier (dir.), Agir ensemble : l'action didactique conjointe du professeur et des élèves (p. 13-49). Rennes : Presses universitaires de Rennes.

Sensevy, G. (2011). Le sens du savoir. Éléments pour une théorie de l'action conjointe en didactique. Bruxelles : de Boeck.

Sensevy, G. (2014). Characterizing teaching effectiveness in the Joint Action Theory in Didactics : an exploratory study in primary school. Journal of Curriculum Studies, 46(5), 577-610, [en ligne : https://doi.org/10.1080/002 20272.2014.931466]

Sinclair, J. M. H., \& Coulthard, R. M. (1975). Towards an analysis of discourse. London: Open University Press.

Sinclair, J. M. H., \& Coulthard, R. M. (1992). Towards an analysis of discourse. In M. Coulthard (Ed.), Advances in spoken discourse (p. Ch. 1). London : Routledge.

Tiberghien, A., Malkoun, L., Buty, C., Souassy, N., \& Mortimer, E. (2007). Analyse des savoirs en jeu en classe de physique à différentes échelles de temps. In G. Sensevy \& A. Mercier (Éds.), Agir ensemble : Éléments de théorisation de l'action conjointe du professeur et des élèves (p. 93-122). Rennes: PUR.

Tiberghien, A., \& Malkoun, L. (2007). Différenciation des pratiques d'enseignement et acquisitions des élèves du point de vue du savoir. Éducation et Didactique, 1, 29-54.

Tiberghien, A. \& Venturini, P. (2015). Articulation des niveaux microscopiques et mésoscopiques dans les analyses de pratiques de classe à partir de vidéos. Recherches en Didactique des Sciences et des Technologies, 11, 53-78.

Tiberghien, A., \& Venturini, P (2018). Characterisation of the didactic contract using the video of the classroom as main data. In L. Xu, G. Aranda \& D. Clarke, Video-based Research in Education : Cross-disciplinary Perspectives. (p. 158-175). London \& New York : Routledge.

Venturini, P., \& Tiberghien, A. (2012). Mise en œuvre de la démarche d'investigation dans le cadre des nouveaux programmes de sciences physiques et chimiques : étude de cas au collège. Revue Française de Pédagogie, 180, 95-120.

Vince, J., Miguet, A.-M., Perrey, S., \& Tiberghien, A. (2016). Structurer son enseignement à l'aide d'activités Quelle place et quelle forme pour l'institutionnalisation ? Le BUP (Bulletin de l'Association Des Professeurs de Physique et de Chimie), 110(988), 1305-1325.

Yackel, E., \& Cobb, P. (1996). Sociomathematical Norms, Argumentation, and Autonomy in Mathematics. Journal for Research in Mathematics Education, 27(4), 458-477.

Wittgenstein, L., \& Anscombe, G. E. M. (1997). Philosophical investigations. Oxford, UK ; Malden, Mass. : Blackwell. 


\section{ANNEXE 1 : DOCUMENT ASSOCIÉ À L'ACTIVITÉ 4 (ÉNONCÉ DE LA TÂCHE)}

\section{Étude de l'électrocardiogramme}

Objectifs : déterminer graphiquement une période et trouver la fréquence.

Lélectrocardiogramme (ECG) est un examen fondamental en cardiologie. Il permet de diagnostiquer des troubles du rythme cardiaque, des infarctus, etc. Ces troubles peuvent être dues à des malformations ou à des habitudes alimentaires ...

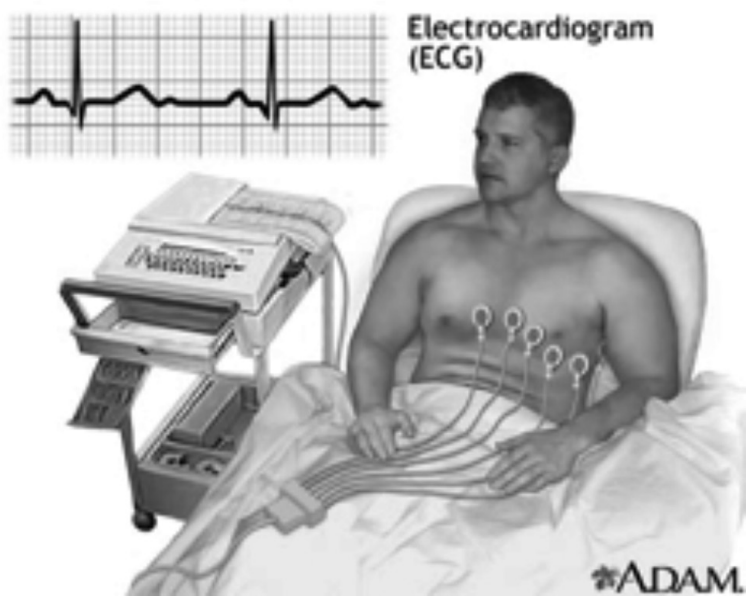

Il s'agit d'un examen indolore, réalisé en quelques minutes, en plaçant des électrodes sur le thorax, les bras et les jambes. Ces électrodes mesurent une tension électrique dont la valeur dépend de l'état de contraction du cœur. L'évolution de cette tension au cours du temps est affichée sous forme de courbe (voir ci-dessous ECG 1 et ECG 2).
ECG du patient $n^{\circ} 1$

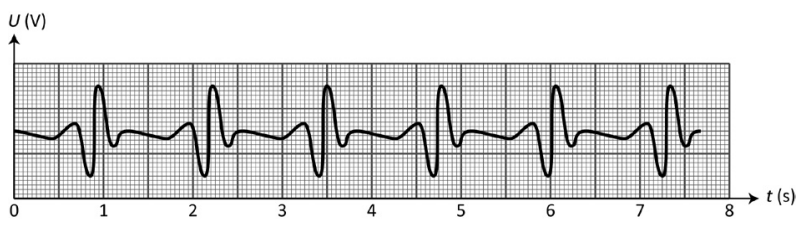

ECG du patient $n^{\circ} 2$

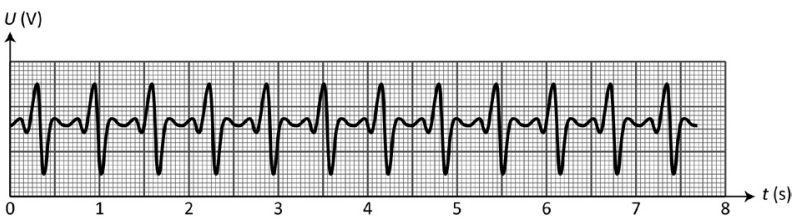

- Quel phénomène périodique est décrit par un ECG?

- Sur un ECG, quelle grandeur physique est représentée en abscisse? en ordonnée?

- Repasser en couleur un motif du signal périodique.

- Parmi fréquence et période, quelle est la grandeur qu'on peut repérer sur un ECG? Représenter cette grandeur sur chaque ECG.

- Sans faire de calcul, indiquer l'ECG qui correspond à la plus grande fréquence cardiaque. Quel enregistrement a été réalisé avec un patient au repos?

- Mesurer en seconde la période des battements du cour du patient $n^{\circ} 2$.

- En déduire sa fréquence cardiaque en $\mathrm{Hz}$ puis en batt/min. 


\section{ANNEXE 2 : ÉPISODES DE NIVEAU N-1 DU JEU S4 J9}

Le jeu S4 J9 intitulé « En collaborant avec son partenaire, exploiter deux ECG pour identifier et déterminer ses grandeurs caractéristiques (fréquence cardiaque période) » a été structuré en 23 épisodes, présentés ci-dessous. Le travail dans ce jeu se déroule en petits groupes et concerne l'activité 4

Tableau 18: Épisodes de niveau N-1 relatifs au jeu S4 J9 Exploiter en groupes de deux des électrocardiogrammes en identifiant et déterminant les grandeurs concernées - fréquence et période ${ }^{15}$

\begin{tabular}{|c|c|c|c|c|}
\hline $\begin{array}{l}\text { Épisode de } \\
\text { niveau N-1 }\end{array}$ & Titre & Début-Fin & Durée & $\begin{array}{l}\text { Épisode } \\
\text { centré sur } \\
\text { le savoir }\end{array}$ \\
\hline Épisode a & Mise au travail & $\begin{array}{l}0: 47: 38.5 \\
0: 47: 56.9\end{array}$ & 0:00:18 & \\
\hline Épisode b & Différenciation unité (V) de la grandeur physique (tension) & $\begin{array}{l}0: 47: 56.9 \\
0: 48: 34.9\end{array}$ & 0:00:41 & 1 \\
\hline Épisode c & Identification de la grandeur tension sur le graphe & $\begin{array}{l}0: 48: 34.9 \\
0: 49: 53.1\end{array}$ & 0:01:18 & 1 \\
\hline Épisode d & Rectification d'un élément de l'énoncé de l'activité & $\begin{array}{l}0: 49: 53.1 \\
0: 50: 37.4\end{array}$ & $0: 00: 44$ & \\
\hline Épisode e & $\begin{array}{l}\text { Explicitation du terme "repérer" dans T ou f? quelle grandeur peut-on } \\
\text { repérer }\end{array}$ & $\begin{array}{l}0: 50: 37.4 \\
0: 51: 17.8\end{array}$ & 0:00:40 & 1 \\
\hline Épisode f & Explicitation du terme grandeur & $\begin{array}{l}0: 51: 17.8 \\
0: 53: 31.3\end{array}$ & $0: 02: 13$ & 1 \\
\hline Épisode $g$ & $\begin{array}{l}\text { Signalement de deux réponses inadaptées: représentation de } \mathrm{T} \text { ou f sur } \\
\text { l'ECG et mesure de T }\end{array}$ & $\begin{array}{l}0: 53: 31.3 \\
0: 55: 04.8\end{array}$ & 0:01:33 & 1 \\
\hline Épisode $\mathrm{h}$ & Invitation à travailler & $\begin{array}{l}0: 55: 04.8 \\
0: 56: 04.4\end{array}$ & 0:01:00 & \\
\hline Épisode i & $\begin{array}{l}\text { Signalement d'une réponse inadaptée (quel phénomène périodique est } \\
\text { décrit) }\end{array}$ & $\begin{array}{l}0: 56: 15.3 \\
0: 58: 22.8\end{array}$ & $0: 02: 07$ & 1 \\
\hline Épisode j & Explication de termes de l'énoncé (parmi et et f, représenter la grandeur) & $\begin{array}{l}0: 58: 46.8 \\
0: 59: 47.0\end{array}$ & 0:01:00 & 1 \\
\hline Épisode $\mathrm{k}$ & Discussion argumentée sur la grandeur repérable ( $\mathrm{T}$ ou f) sur l'ECG & $\begin{array}{l}0: 59: 47.0 \\
1: 01: 32.4\end{array}$ & $0: 01: 45$ & 1 \\
\hline Épisode 1 & Identification du phénomène périodique & $\begin{array}{l}1: 01: 32.4 \\
1: 03: 03.8\end{array}$ & $0: 01: 31$ & 1 \\
\hline Épisode $\mathrm{m}$ & $\begin{array}{l}\text { Suite de la discussion sur la représentation de la grandeur T Invitation à } \\
\text { repérer une période sur l'ECG }\end{array}$ & $\begin{array}{l}1: 03: 03.8 \\
1: 03: 24.7\end{array}$ & $0: 00: 21$ & 1 \\
\hline Épisode $n$ & Discussion sur phénomène périodique & $\begin{array}{l}1: 03: 30.1 \\
1: 04: 01.9\end{array}$ & 0:00:32 & 1 \\
\hline Épisode o & Opérationnalisation de la notion de période sur le graphe & $\begin{array}{l}1: 04: 08.1 \\
1: 05: 25.8\end{array}$ & 0:01:18 & 1 \\
\hline Épisode $\mathrm{p}$ & $\begin{array}{l}\text { Discussion sur la distinction entre repérage du motif et repérage de la } \\
\text { période sur l'ECG }\end{array}$ & $\begin{array}{l}1: 06: 10.5 \\
1: 07: 37.5\end{array}$ & 0:01:27 & 1 \\
\hline Épisode q & Remise au travail & $\begin{array}{l}1: 07: 37.5 \\
1: 07: 41.3\end{array}$ & 0:00:03 & \\
\hline Épisode $\mathrm{r}$ & Discussion sur lecture de la période sur l'ECG & $\begin{array}{l}1: 07: 41.3 \\
1: 08: 30.0\end{array}$ & 0:00:49 & 1 \\
\hline Épisode s & $\begin{array}{l}\text { Discussion sur repérage période fréquence sur ECG et distinction repé- } \\
\text { rage motif période }\end{array}$ & $\begin{array}{l}1: 08: 30.0 \\
1: 09: 28.6\end{array}$ & 0:00:59 & 1 \\
\hline Épisode $\mathrm{t}$ & Prise de connaissance sans commentaire & $\begin{array}{l}1: 09: 28.6 \\
1: 09: 36.8\end{array}$ & 0:00:08 & \\
\hline Épisode u & $\begin{array}{l}\text { Discussion sur opérationnalisation définition période sur ECG pour } \\
\text { mesurer }\end{array}$ & $\begin{array}{l}1: 09: 36.8 \\
1: 10: 32.1\end{array}$ & 0:00:55 & 1 \\
\hline Épisode v & Discussion sur la valeur de la période sur ECG (mesure approximative) & $\begin{array}{l}1: 10: 32.1 \\
1: 11: 08.1\end{array}$ & 0:00:36 & 1 \\
\hline \multirow[t]{2}{*}{ Épisode w } & Annonce de la correction collective & $\begin{array}{l}1: 11: 15.0 \\
1: 11: 37.4\end{array}$ & 0:00:22 & \\
\hline & Intervalle & $\begin{array}{l}1: 11: 37.4 \\
1: 11: 41.4\end{array}$ & 0:00:03 & \\
\hline TOTAL & & & & 17 \\
\hline
\end{tabular}

15. Pour distinguer les épisodes déterminés par le passage du professeur dans un groupe (Jeu S4 J9) des épisodes associés à une transaction élémentaire (Jeu S4 J10), nous avons repéré les premiers avec des lettres et les seconds avec des nombres. 


\section{ANNEXE 3 : ÉPISODES DE NIVEAU N-1 DU JEU S4 J10}

Le jeu S4 J10 intitulé « Comprendre grâce à la discussion collective la correction de la détermination de la fréquence et la période de deux ECG » a été structuré en 7 épisodes, présentés ci-dessous. Le travail dans ce jeu se déroule en classe entière et concerne l'activité 4

Tableau 19 : Épisodes de niveau N-1 relatifs au jeu S4 J10

(Comprendre et écrire la correction de l'exploitation d'électrocardiogrammes en classe entière).

\begin{tabular}{|c|c|c|c|c|}
\hline Épisodes & Titre & Début-Fin & Durée & $\begin{array}{c}\text { Épisode } \\
\text { Savoir }\end{array}$ \\
\hline Épisode 1 & Nature du phénomène périodique en jeu dans l'ECG & $\begin{array}{l}1: 11: 41.4 \\
1: 13: 15.9\end{array}$ & 0:01:34 & 1 \\
\hline Épisode 2 & Grandeurs représentées sur l'ECG (U et t) & $\begin{array}{l}1: 13: 15.9 \\
1: 14: 24.9\end{array}$ & 0:01:09 & 1 \\
\hline Épisode 3 & Correction de l'identification du motif & $\begin{array}{l}1: 14: 24.9 \\
1: 16: 36.5\end{array}$ & 0:02:11 & 1 \\
\hline Épisode 4 & Identification de la grandeur ( $\mathrm{T}$ ou F) lue directement sur l'ECG & $\begin{array}{l}1: 16: 36.5 \\
1: 21: 01.7\end{array}$ & $0: 04: 25$ & 1 \\
\hline Épisode 5 & Comparaison des fréquences des 2 ECG & $\begin{array}{l}1: 21: 01.7 \\
1: 23: 42.5\end{array}$ & $0: 02: 40$ & 1 \\
\hline Épisode 6 & Détermination de la valeur de T sur l'ECG & $\begin{array}{l}1: 23: 42.5 \\
1: 24: 42.4\end{array}$ & 0:00:59 & 1 \\
\hline Épisode 7 & Calcul de la fréquence en $\mathrm{Hz}$ et en battements/minute & $\begin{array}{l}1: 24: 42.4 \\
1: 26: 31.9\end{array}$ & 0:01:49 & 1 \\
\hline
\end{tabular}

